\title{
The meso-structured magnetic atmosphere
}

\section{A stochastic polarized radiative transfer approach ${ }^{\star}$}

\begin{abstract}
T. A. Carroll and M. Kopf
Astrophysikalisches Institut Potsdam, An der Sternwarte 16, 14482 Potsdam, Germany

e-mail: tcarroll@aip.de

Received 19 December 2006 / Accepted 16 March 2007

ABSTRACT

We present a general radiative transfer model which allows the Zeeman diagnostics of complex and unresolved solar magnetic fields. Present modeling techniques still rely to a large extent on a-priori assumptions about the geometry of the underlying magnetic field. In an effort to obtain a more flexible and unbiased approach we pursue a rigorous statistical description of the underlying atmosphere. Based on a Markov random field model the atmospheric structures are characterized in terms of probability densities and spatial correlations. This approach allows us to derive a stochastic transport equation for polarized light valid in a regime with an arbitrary fluctuating magnetic field on finite scales. One of the key ingredients of the derived stochastic transfer equation is the correlation length which provides an additional degree of freedom to the transport equation and can be used as a diagnostic parameter to estimate the characteristic length scale of the underlying magnetic field.

It is shown that the stochastic transfer equation represents a natural extension of the (polarized) line formation under the microand macroturbulent assumption and contains both approaches as limiting cases. In particular, we show how in an inhomogeneous atmosphere asymmetric Stokes profiles develop and that the correlation length directly controls the degree of asymmetry and net circular polarization (NCP). In a number of simple numerical model calculations we demonstrate the importance of a finite correlation length for the polarized line formation and its impact on the resulting Stokes line profiles.
\end{abstract}

Key words. radiative transfer - line: formation - line: profiles - Sun: photosphere - Sun: magnetic fields - polarization

\section{Introduction}

The entire solar photosphere exhibits a rich structure of large and small scale magnetic features like sunspots, pores, faculae or plages. But except for sunspots and pores these magnetic fields are not spatially resolved with present telescopes, although these fields clearly manifest themselves in high resolution spectropolarimetric observations. With the improvement of spectropolarimetric sensitivity and spatial resolution over the last years it became clear that these unresolved magnetic fields are much more ubiquitous than previously thought. This raises the question of the significance of these elusive and complex magnetic fields for the solar magnetism in general (Schrijver \& Title 2003; Sánchez Almeida 2004) and how these magnetic fields can be appropriately investigated by means of spectropolarimetric observations and Zeeman diagnostics.

The concept of magnetic flux tubes as the building blocks of solar surface magnetism has surely played a key role in our understanding of the unresolved magnetic field of the solar photosphere (Stenflo 1976). A wealth of diagnostic techniques based on the flux tube concept have been developed over the years and greatly improved our physical insight into the nature of the photospheric magnetism (e.g. Solanki 1993). The interpretation of the Zeeman induced spectral line polarization in the context of the flux tube model rests on the idea of tube-like magnetic structures surrounded by an effectively non-magnetic atmosphere. A

* Appendices are only available in electronic form at http://www. aanda.org so called 1.5-dimensional radiative transfer model is then applied where a number of rays (line-of-sights) piercing through the 2- or 3-dimensional geometry of the model atmosphere in order to obtain its spectral signature (Stenflo 1994). From the standpoint of radiative transfer this approach provides a rather macroscopic treatment of the problem, as each line-of-sight possesses its own individual atmospheric structure and the averaging process for all line-of-sights (LOS) is performed after the actual integration of the transfer equation. Another problem here is, a relatively detailed knowledge about the number density and the geometrical arrangement of the flux tubes are needed in advance.

A quite different approach to characterize the small scale and unresolved nature of the photospheric magnetic field was put forward by Sanchez Almeida et al. (1996). In an attempt to reconcile the ubiquity of asymmetric Stokes $V$ profiles with the underlying magnetic field structure, they postulated the microstructured magnetic atmosphere (MISMA). In that model the degree of fluctuation and intermittency is much higher than in the classical flux tube picture and the magnetic field is assumed to be structured over scales much smaller than the mean free path of photons. This approach could successfully reproduce many of the observed Stokes $V$ profile asymmetries in quiet and active solar regions (Sanchez Almeida 1997; Sánchez Almeida \& Lites 2000). In terms of radiative transfer modeling the MISMA approximation is a statistical approach which requires that the photons undergo a rapid and random fluctuation on smallest scales along their trajectory (line-of-sight). If the fluctuation rate per length scale is much higher than the (true) absorption rate this 
scenario represents the classical microturbulent approach and allows a simplified treatment of the radiative transfer. The statistical averaging process (convolution) over all atmospheric structures can then be performed before the actual integration of the transfer equation. Despite its appealing simplicity in the way this approach treats the radiative transfer the idealized assumptions about the underlying atmosphere strongly limits the application of this approach. As soon as only one of the ensemble structures exceeds the micro-scale criteria (and we will see later that this happens at surprisingly small scales) the microturbulent approximation fails. Moreover, magnetic microstructures can not in general be describe in the microturbulent limit. As elongated thin magnetic structures close to equipartition have a preferential vertical alignment due to buoyancy forces the path length of an individual line-of-sight that traverse through such a magnetic structure depends on the angle between the line-of-sight and the axis of the structure. The same structures that manifest themselves as microstructures in observations near the solar limb can become macrostructures in disk-center observations. This simply reflects the fact that the vertical and horizontal dimension of flux structures in the solar photosphere are very different.

Over the last decade numerical magneto-convection simulations have reached a level of realism where they can provide valuable information about the three-dimensional structure of the photospheric plasma (Stein \& Nordlund 1998; Steiner et al. 1998; Schüssler 2003; Vögler \& Schüssler 2003; Vögler et al. 2005; Schaffenberger et al. 2005; Stein \& Nordlund 2006). Recent magneto-convection simulations of plage like regions have shown that flux expulsion and convective field amplification may lead to structures in the supergranular network which resemble thin magnetic flux tubes or flux sheets (Vögler et al. 2005). For the quiet sun, numerical simulations indicate a lesser degree of organization with more fragmented and incoherent structures (Schaffenberger et al. 2005; Stein \& Nordlund 2006). These simulations suggests that neither a predefined static macrostructured nor a pure microturbulent approach is an appropriate starting point for the interpretation of spectropolarimetric observations. The magnetic field seems to comprise a broad range of structures which span from micro- (over meso-) to macro-scales. This is picture is supported by recent Zeeman and Hanle based diagnostics which are consistent with a continuous magnetic field strength and flux distribution (Khomenko et al. 2003; Dominguez Cerdeña et al. 2003b; Trujillo Bueno et al. 2004).

So the present paper is an attempt to enhance the diagnostic capabilities of the Zeeman effect and to bridge the gap between the macro- and microstructured paradigm to obtain a more general mesostructured approach. Our approach is based on a statistical description of the underlying atmosphere and its relevant parameters in terms of a Markov random field. One of the aims is to explicitly incorporate the spatial coherences and to account for the finite character of the underlying magnetic field. Although the model atmosphere is inherently non-plane parallel this approach allows us to formulate the transfer of polarized light with the help of a one dimensional stochastic transport equation.

The idea of a stochastic radiative transfer approach is by no means new, several attempts were made to describe the line formation in random velocity fields, for example Auvergne et al. (1973), Gail et al. (1974), Frisch \& Frisch (1976), Magnan (1985), Gu et al. (1995), Nikoghossian et al. (1997) The literature for polarized line formation in stochastic magnetic media is considerably shorter, there are only a few attempts known to the authors by Domke \& Pavlov (1979), Landi Degl'Innocenti \& Landolfi (2004) and very recently by Carroll \& Staude (2003), Carroll \& Staude (2005a), Carroll \& Staude (2005b), Carroll \& Staude (2006) and Frisch et al. (2005), Frisch et al. (2006). The latter authors nicely demonstrated how the finite character of the underlying structures affect the line formation. But unlike most of the aforementioned authors we will not consider the solution under some limiting aspects, our intention is rather to derive a general statistical description of the magnetized atmosphere and then, using this description, to derive a (stochastic) transfer equation for polarized light.

This paper is organized as follows: in Sect. 2 we introduce the general statistical concept of the mesostructured magnetic atmosphere. A model is presented which explicitly takes into account the spatial coherency of the atmospheric parameters by means of a Markov random field. Based on that description, we derive in Sect. 3 the stochastic transport equation for polarized light. We present a formal solution in terms of a modified evolution operator and show that the microturbulent and the macroturbulent approximations are special limits of our more general mesostructured approach. In Sect. 3 we also show that the origin of asymmetric Stokes profiles and a net-circularpolarization (NCP) are natural consequences in a mesostructured magnetic atmosphere and that the degree of asymmetry depends directly on the characteristic length scale (correlation length) of the underlying magnetic structures. In Sect. 4 we give a brief description of the numerical realization of our stochastic approach. Then a number of numerical experiments follow, where we demonstrate how the arrangement of the underlying atmosphere and in particular the correlation length of the magnetic structures have a decisive impact on the line formation process and on the appearance of the resulting Stokes profiles. Section 5 finally gives a brief summary and draws the main conclusions.

\section{The Mesostructured magnetic atmosphere}

Due to solar convection we know that the photospheric plasma has a rather complicated structure in terms of its temperature and velocity distribution. This dynamic behavior also influences the magnetic field, in particular in a regime where the plasma $\beta$ is close to one. It is this complexity why we have chosen to pursue a rigorous statistical approach to describe the atmosphere and the underlying magnetic field.

In the following we will present a discrete description of the atmosphere, following the concept of numerical magnetoconvective simulations, we approximate the atmospheric structure by a discrete 3 -dimensional lattice structure with a regular arrangement of nodes. To each node we will assign a number of physical parameters (a state vector) which describe the physical conditions at the particular locations of the nodes. Each node is moreover associated with a multivariate probability density function and a neighborhood relation. This neighborhood relation will be given in terms of a Markov model which then allows us to derive, for an arbitrary trajectory or line-of-sight a differential equation which describes the evolution of the probability density function. This differential equation forms the basis for Sect. 3, where we describe the line formation as a stochastic process and derive the stochastic transport equation for polarized light. 


\subsection{The atmosphere as a Markov random field}

Let us begin by introducing the state vector $\boldsymbol{B}$ for an arbitrary position (node) in the atmosphere as a vector quantity which includes all relevant atmospheric parameters,

$\boldsymbol{B}^{*}=\left(\alpha_{1}^{*}, \alpha_{2}^{*}, \ldots \alpha_{n}^{*}\right)^{T}$.

The vector $\boldsymbol{B}$ shall contain atmospheric parameters like temperature, velocity, magnetic field etc.. We assume, that the parameters $\alpha_{n}$ are random variables, hence the vector $\boldsymbol{B}$ represents a multivariate random variable or a random vector respectively, for which we define a probability density function (pdf) of the form

$p(\boldsymbol{B})=p\left(\alpha_{1}, \alpha_{2}, \ldots \alpha_{n}\right)$,

where the probability $P(\boldsymbol{B} \leq \hat{\boldsymbol{B}} \leq \boldsymbol{B}+\mathrm{d} \boldsymbol{B})$ of finding a realization of $\hat{\boldsymbol{B}}$ within $\boldsymbol{B} \leq \hat{\boldsymbol{B}} \leq \boldsymbol{B}+\mathrm{d} \boldsymbol{B}$ is

$$
\begin{aligned}
P(\boldsymbol{B} \leq \hat{\boldsymbol{B}} \leq \boldsymbol{B}+\mathrm{d} \hat{\boldsymbol{B}}) & =p\left(\alpha_{1}, \ldots, \alpha_{n}\right) \mathrm{d} \alpha_{1} \ldots \mathrm{d} \alpha_{n} \\
& =p(\boldsymbol{B}) \mathrm{d} \boldsymbol{B},
\end{aligned}
$$

and which satisfy the following normalization condition:

$p(\boldsymbol{B}) \geq 0$ and $\int_{-\infty}^{\infty} p(\boldsymbol{B}) \mathrm{d} \boldsymbol{B}=1$.

Here, we have assumed that $\boldsymbol{B}$ is a real-valued random vector and we want to keep this notation in the following without loss of generality. For the sake of brevity and clarity, we also will not distinguish between the stochastic variable and its realizations (states) hereafter, furthermore the integration limits will be omitted as long as the integration is performed over the entire domain or state space respectively.

As mentioned in the introduction, we will now consider a spatially discretized approximation of the atmosphere in terms of a three dimensional uniform grid structure. The grid, hereafter called the random field, is made up by a number of grid points (nodes) where each node is associated with a random atmospheric vector $\boldsymbol{B}_{i}$, and its respective probability density function

$p_{s_{i}}\left(\boldsymbol{B}_{i}\right)=p\left(\boldsymbol{B}_{i}, s_{i}\right)=p\left(\alpha_{i_{1}}, \alpha_{i_{2}}, \ldots \alpha_{i_{n}}, s_{i}\right)$.

The index $s_{i}$ specifies the spatial position of the node. In order to obtain a general probabilistic description of the entire field, we need to take into account that the random vectors $\boldsymbol{B}_{i}$ at their specific positions $s_{i}$ might be correlated among each other. The statistical state of the grid is therefore described in its most general form by a joint probability density for the entire field $p\left(\boldsymbol{B}_{1}, s_{1} ; \ldots ; \boldsymbol{B}_{n}, s_{n}\right)$. This joint pdf or $n$-point distribution function can also be expressed in terms of the respective conditional probability density function

$$
\begin{aligned}
p\left(\boldsymbol{B}_{1}, s_{1} ; \ldots ; \boldsymbol{B}_{n}, s_{n}\right)= & p\left(\boldsymbol{B}_{1}, s_{1} \mid \boldsymbol{B}_{2}, s_{2} ; \ldots ; \boldsymbol{B}_{n}, s_{n}\right) \\
& \times p\left(\boldsymbol{B}_{2}, s_{2} ; \ldots ; \boldsymbol{B}_{n}, s_{n}\right),
\end{aligned}
$$

which emphasizes the spatial dependency (correlation) of the field. The state vector $\boldsymbol{B}_{1}$ at $s_{1}$ will in general depend on other state vectors within a certain region or radius around $s_{1}$. In general it will be difficult to formulate an approximation for the joint probability density function or conditional probability density (6) in terms of an appropriate n-point correlation function. To reduce the complexity of the random field the simplest assumption is that of a completely uncorrelated field. Thus, the realization of a state vector $\boldsymbol{B}_{i}$ at the position $s_{i}$ is completely independent and uncorrelated from all other realizations at other positions. With this assumption the joint probability density (6) factorizes into the products of the individual one-point probability densities of the respective state vectors,

$$
\begin{aligned}
p\left(\boldsymbol{B}_{1}, s_{1} ; \ldots ; \boldsymbol{B}_{n}, s_{n}\right) & =p\left(\boldsymbol{B}_{1}, s_{1}\right) p\left(\boldsymbol{B}_{2}, s_{2}\right) \ldots p\left(\boldsymbol{B}_{n}, s_{n}\right) \\
& =\prod_{i}^{n} p\left(\boldsymbol{B}_{i}, s_{i}\right) .
\end{aligned}
$$

This description of the atmosphere in terms of a spatially uncorrelated field where the atmospheric state vectors $\boldsymbol{B}_{i}$ are exclusively described by their respective one-point pdf, would immediately lead us to a microturbulent approach. Our intention here is, however, to go beyond this microturbulent concept in order to take - at least to the lowest order - correlation effects into account. In the following we will therefore consider the particular type of a Markovian field structure. A Markov random field is characterized by a rather simple neighborhood relation which describes the spatial correlation of each node in the field with its neighboring nodes. The neighborhood $N_{i}$ at the position $s_{i}$ can be defined as follows

$N_{i} \equiv\left\{s_{j}:\left\|s_{i}-s_{j}\right\| \leq r\right\}$,

where $r$ gives the spatial distance or radius between $s_{i}$ and the neighboring points $s_{j}$. The Markov property of the random field can then be defined such that for each state vector $\boldsymbol{B}_{i}$ at $s_{i}$ the following conditional probability density function holds

$p\left(\boldsymbol{B}_{i}, s_{i} \mid \boldsymbol{B}_{j}, s_{j}\right) ;$ where $i \neq j$ and $s_{j} \in N_{i}$.

The conditional probability of the state vector $\boldsymbol{B}_{i}$ is thus only correlated to its neighboring states. The underlying assumption here is that all higher order spatial correlations are sufficiently small (and rapidly decay to zero) compared to first order correlation effects such that the atmospheric vector $\boldsymbol{B}_{i}$ at $s_{i}$ decouples from situations (states) which are further away than the immediate neighborhood $N_{i}$.

\subsection{A sample path through the Markov field}

In this work we will concentrate on the radiative transfer in a non-scattering atmosphere and therefore let us consider a particular trajectory - a sample path - through the above described random field. The sample path is described by a onedimensional, unidirectional and contiguous sequence (path or trajectory) with $s_{1}<s_{2}<\ldots<s_{n}$ through the random Markov field, where $s_{i}$ now determines the node position along our sample path. Note, that the state space vector $\boldsymbol{B}$ can either be discrete or continuous, without loss of generality and for mathematical convenience we assume in the following a continuous state space. Along the trajectory we encounter a sequence of realizations with $\boldsymbol{B}_{1}, s_{1} ; \boldsymbol{B}_{2}, s_{2} ; \ldots ; \boldsymbol{B}_{n}, s_{n}$ where the random vector $\boldsymbol{B}_{i}$ in general adopts different values at different positions $s_{i}$. This stochastic sequence along the trajectory defines a stochastic process where the state vector $\boldsymbol{B}$ has a distinct dependence on the spatial position $s$. From the Markov property of the random field we know that there exist a conditional probability density for the vector $\boldsymbol{B}_{i}$ at each position $s_{i}$ in the atmosphere which relates $\boldsymbol{B}_{i}$ to the state vectors of the neighborhood. For the stochastic process along our sample path the three dimensional neighborhood at each position reduces to a one dimensional relation. In particular for our unidirectional path this means that the state $\boldsymbol{B}_{i}$ at $s_{i}$ is correlated only to its predecessor state $\boldsymbol{B}_{i-1}$ at $s_{i-1}$. This represents the typical property of a Markov process 
(van Kampen 1992) and allows us to write for the conditional probability densities at $s_{n}$

$p\left(\boldsymbol{B}_{n}, s_{n} \mid \boldsymbol{B}_{n-1}, s_{n-1} ; \ldots ; \boldsymbol{B}_{1}, s_{1}\right)=p\left(\boldsymbol{B}_{n}, s_{n} \mid \boldsymbol{B}_{n-1}, s_{n-1}\right)$,

where $s_{n}$ and $s_{n-1}$ are neighboring points along our sample path. The conditional probability which, in the framework of Markov processes, is also called transition probability expresses the property that the probability for a transition from the state $\boldsymbol{B}_{s_{n-1}}$ at $s_{n-1}$ to a state $\boldsymbol{B}_{n}$ at $s_{n}$ only depends on the condition (state) at $s_{n-1}$ and not on the (spatial) history prior to $\boldsymbol{B}_{n-1}$. As can be shown, the following equation must hold for a Markov process (van Kampen 1992),

$$
\begin{aligned}
& p\left(\boldsymbol{B}_{3}, s_{3} \mid \boldsymbol{B}_{1}, s_{1}\right)= \int p\left(\boldsymbol{B}_{3}, s_{3} \mid \boldsymbol{B}_{2}, s_{2}\right) \\
& \times p\left(\boldsymbol{B}_{2}, s_{2} \mid \boldsymbol{B}_{1}, s_{1}\right) \mathrm{d} \boldsymbol{B}_{2},
\end{aligned}
$$

where the integration is performed over the entire state space. This is the Chapman-Kolmogorov equation which states that the transition probability between two states $\boldsymbol{B}_{1}$ and $\boldsymbol{B}_{3}$ with $s_{1}<s_{3}$ corresponds to the product of the transition probability between the initial state $\boldsymbol{B}_{1}$ and some intermediate state $\boldsymbol{B}_{2}$ as well as the transition from this intermediate state to the final state $\boldsymbol{B}_{3}$, integrated over all possible intermediate states.

In the following, we will concentrate on a homogeneous stochastic process. A homogeneous process is characterized by a transition probability $p\left(\boldsymbol{B}_{2}, s_{2} \mid \boldsymbol{B}_{1}, s_{1}\right)$ that only depends on the spatial distance $\Delta s=s_{2}-s_{1}$ between the two states and not on their particular spatial positions. We therefore omit the indices and consider, without loss of generality, $s$ as a continuous parameter.

Let us now define a transition probability which obeys the Chapman-Kolmogorov Eq. (11). To obtain such a model we have to make assumptions about the underlying (magnetic) structure of the atmosphere and how this structure determines the short scale behavior of the transition probability. Following the idea of thin magnetic flux tubes we can assume that the photosphere is characterized by sharp transitions between individual magnetic structures and their non-magnetic surrounding. This picture may be justified by the extreme small boundary layers of thin magnetic flux tubes which are well below the photon mean free path (Schüssler 1986). The Markov process must therefore exhibit a distinct discontinuous character where abrupt jumps between different atmospheric regimes along a trajectory occur. A suitable definition of the transition probability for small $\Delta s$, between two states $\boldsymbol{B}^{\prime}$ and $\boldsymbol{B}^{\prime \prime}$ can be obtained by the following discontinuous process

$$
\begin{aligned}
p\left(\boldsymbol{B}^{\prime \prime}, s+\Delta s \mid \boldsymbol{B}^{\prime}, s\right)= & \mathrm{e}^{-\gamma\left(\boldsymbol{B}^{\prime}\right) \Delta s} \delta\left(\boldsymbol{B}^{\prime}-\boldsymbol{B}^{\prime \prime}\right) \\
& +\left(1-\mathrm{e}^{-\gamma\left(\boldsymbol{B}^{\prime}\right) \Delta s}\right) p\left(\boldsymbol{B}^{\prime \prime}\right),
\end{aligned}
$$

where $\gamma\left(\boldsymbol{B}^{\prime}\right)$ is the fluctuation rate of the atmospheric structure $\boldsymbol{B}_{s}$ and $p\left(\boldsymbol{B}^{\prime \prime}\right)$ the spatial independent (stationary) probability density. The fluctuation rate can be expressed with the help of the correlation length $\lambda$ to give

$\gamma\left(\boldsymbol{B}_{s}\right)=\lambda^{-1}\left(\boldsymbol{B}_{s}\right)$

As can be seen from Eq. (12) the correlation length can be considered as the mean length scale of the structures. The process (12), which is sometimes called Kubo-Anderson process (Frisch \& Frisch 1976) describes how the probability of the state vector $\boldsymbol{B}^{\prime \prime}$ depends on the spatial distance $\Delta s$ between $\boldsymbol{B}^{\prime \prime}$ and the initially known vector $\boldsymbol{B}^{\prime}$. The probability for staying in the initial state or atmospheric regime $\boldsymbol{B}^{\prime}$ decreases exponentially with the distance $\Delta s$ while the probability for a transition to another atmospheric regime $\boldsymbol{B}^{\prime \prime}$ increases with $\Delta s$ and is weighted by the overall probability of the state $p\left(\boldsymbol{B}^{\prime \prime}\right)$. As the conditional probability density of the process depends on the spatial distance $\Delta s$ we can use the Kubo-Anderson process (12) and the ChapmanKolmogorov Eq. (11) to derive the following integro-differential equation (see Appendix A) which describes the evolution of the probability density for $\boldsymbol{B}$ along a particular trajectory.

$$
\begin{aligned}
\frac{\partial p(\boldsymbol{B}, s)}{\partial s}= & \int w\left(\boldsymbol{B}, \boldsymbol{B}^{\prime}, s\right) p\left(\boldsymbol{B}^{\prime}, s\right) \mathrm{d} \boldsymbol{B}^{\prime} \\
& -\int w\left(\boldsymbol{B}^{\prime}, \boldsymbol{B}, s\right) p(\boldsymbol{B}, s) \mathrm{d} \boldsymbol{B}^{\prime},
\end{aligned}
$$

where $w$ is the transition rate (transitions per unit length scale) and is defined by (A.2). This equation which is also called the master equation of the process describes how the probability density develops along a given trajectory through the atmosphere. The first term on the r.h.s. represents a source or gain term for the probability, which describes transitions from an arbitrary atmospheric state $\boldsymbol{B}^{\prime}$ to the state $\boldsymbol{B}$ under consideration. The second term on the r.h.s. is a sink or loss term for the probability density and describes transitions from the state $\boldsymbol{B}$ to other available atmospheric states. The master equation forms the basis for the following derivation of the stochastic transfer equation for polarized light.

\section{The stochastic transport equation for polarized light}

\subsection{Derivation of the stochastic transport equation}

We now consider a particular photon trajectory along the lineof-sight in a non-scattering atmosphere, which will allow us to describe the radiative transfer in terms of a stochastic process. We begin by writing down the (deterministic) transport equation for polarized light along the ray path coordinate $s$ in a plane parallel atmosphere for a frequency $v$ which is given by

$\frac{\mathrm{d} \boldsymbol{I}_{v}}{\mathrm{~d} s}=\mathbf{K}_{v} \boldsymbol{I}_{v}+\boldsymbol{j}_{v}$

where $\boldsymbol{I}$ is the 4-dimensional Stokes vector $\boldsymbol{I}=I, Q, U, V^{T}$ which describes the intensity and polarization state of the light (e.g. Stenflo 1994). For clarity, we will omit the frequency indices in the following. The absorption matrix $\mathbf{K}$ and the emission vector $\boldsymbol{j}$ are given by

$\mathbf{K}=\left(\begin{array}{cccc}\eta_{I} & \eta_{Q} & \eta_{U} & \eta_{V} \\ \eta_{Q} & \eta_{I} & \rho_{V} & -\rho_{U} \\ \eta_{U} & -\eta_{V} & \eta_{I} & \rho_{Q} \\ \eta_{V} & \rho_{U} & -\rho_{Q} & \eta_{I}\end{array}\right)$

and

$\boldsymbol{j}=\left(j_{I}, j_{Q}, j_{U}, j_{V}\right)^{T}=\left(\eta_{I}, \eta_{Q}, \eta_{U}, \eta_{V}\right)^{T} B$,

where $T$ denotes the transpose of the emission vector and $B$ stands for the Planck function. Here we have assumed that the emission originates under local thermodynamic equilibrium (LTE). For the standard case of a spectral line formed in the presence of a magnetic field, the detailed expression for the entries of the absorption matrix $\mathbf{K}$ as well as for the emission vector $\boldsymbol{j}$ can be found for instance in Rees (1987).

As our model atmosphere is described in statistical terms the observable quantity of interest in our case is now given by the 
mean value of the Stokes vector. We therefore define the mean Stokes vector by the first moment of the probability density function $p(\boldsymbol{I}, \boldsymbol{B}, s)$. Two things are noteworthy here, first, we have to include the spatial position $s$ into the density function since the value of the density function in general depends on the spatial position in the atmosphere since both $\boldsymbol{I}$ and $\boldsymbol{B}$ depend on $s$. Second, we have to include the Stokes vector itself into the statistical description, because the Stokes vector is coupled to the atmospheric conditions via the transport Eq. (15) and therefore the Stokes vector itself becomes a stochastic variable which follows the same stochastic process as the atmospheric vector $\boldsymbol{B}$. The mean Stokes vector at the position $s$ is then given by

$\langle\boldsymbol{I}(s)\rangle=\iint \boldsymbol{I} p(\boldsymbol{I}, \boldsymbol{B}, s) \mathrm{d} \boldsymbol{B} \mathrm{d} \boldsymbol{I}$.

We then separate the joint pdf $p(\boldsymbol{I}, \boldsymbol{B}, s)$ by using the conditional pdf $p(\boldsymbol{I} \mid \mathrm{d} \boldsymbol{B}, s)$ to write Eq. (17) as

$\langle\boldsymbol{I}(s)\rangle=\iint \boldsymbol{I} p(\boldsymbol{I} \mid \boldsymbol{B}, s) p(\boldsymbol{B}, s) \mathrm{d} \boldsymbol{B} \mathrm{d} \boldsymbol{I}$.

The joint pdf can therefore be expressed as the product of the conditional pdf of the Stokes vector $\boldsymbol{I}$ which is conditioned on the atmospheric regime $\boldsymbol{B}$ at $s$ and the structural pdf of the atmospheric vector $\boldsymbol{B}$. We can evaluate the conditional pdf $p(\boldsymbol{I} \mid \mathrm{d} \boldsymbol{B}, s)$ by realizing that this conditional pdf represents the deterministic solution of the transport Eq. (15) under the specific atmospheric conditions $\boldsymbol{B}$. Thus expressing the conditional pdf with the help of the Dirac delta function we obtain

$p(\boldsymbol{I} \mid \boldsymbol{B}, s)=\delta(\boldsymbol{I}-\boldsymbol{I}(\boldsymbol{B}, s))=\delta(\boldsymbol{I}-\hat{\boldsymbol{I}}(s))$,

where $\hat{\boldsymbol{I}}(s)$ is the formal solution of the initial value problem of Eq. (15) given the atmospheric conditions $\boldsymbol{B}$ at $s$. The Dirac delta function of the Stokes vector has the following definition

$\delta(\boldsymbol{I}-\hat{\boldsymbol{I}}(s))=\delta(I-\hat{I}(s)) \delta(Q-\hat{Q}(s)) \delta(U-\hat{U}(s)) \delta(V-\hat{V}(s))$.

Equation (18) can then be written as

$\langle\boldsymbol{I}(s)\rangle=\iint \boldsymbol{I} \delta(\boldsymbol{I}-\hat{\boldsymbol{I}}(s)) p(\boldsymbol{B}, s) \mathrm{d} \boldsymbol{B} \mathrm{d} \boldsymbol{I}$.

Taking the derivative of (18) with respect to the path variable $s$ such that

$\frac{\partial\langle\boldsymbol{I}\rangle}{\partial s}=\frac{\partial}{\partial s} \iint \boldsymbol{I} \delta(\boldsymbol{I}-\hat{\boldsymbol{I}}(s)) p(\boldsymbol{B}, s) \mathrm{d} \boldsymbol{B} \mathrm{d} \boldsymbol{I}$,

and interchanging the differentiation and integration operators we get

$$
\begin{aligned}
\frac{\partial\langle\boldsymbol{I}\rangle}{\partial s}= & \iint\left[\boldsymbol{I}\left(\frac{\partial}{\partial s} \delta(\boldsymbol{I}-\hat{\boldsymbol{I}}(s))\right) p(\boldsymbol{B}, s)\right. \\
& \left.+\boldsymbol{I} \delta(\boldsymbol{I}-\hat{\boldsymbol{I}}(s)) \frac{\partial}{\partial s} p(\boldsymbol{B}, s)\right] \mathrm{d} \boldsymbol{B} \mathrm{d} \boldsymbol{I} .
\end{aligned}
$$

Taking further into account the differential form of the transport Eq. (15), we can write the derivative of the Dirac delta function (20) with respect to the path variable $s$ as

$$
\begin{aligned}
\frac{\partial}{\partial s} \delta(\boldsymbol{I}-\hat{\boldsymbol{I}}(s)) & =\frac{\partial \hat{\boldsymbol{I}}(s)}{\partial s} \nabla_{\hat{\boldsymbol{I}}} \delta(\boldsymbol{I}-\hat{\boldsymbol{I}}(s)) \\
& =\left(-\mathbf{K}_{B} \hat{\boldsymbol{I}}+\boldsymbol{j}_{\boldsymbol{B}}\right) \nabla_{\hat{\boldsymbol{I}}} \delta(\boldsymbol{I}-\hat{\boldsymbol{I}}(s)) \\
& =-\left(-\mathbf{K}_{B} \hat{I}+\boldsymbol{j}_{\boldsymbol{B}}\right) \nabla_{\boldsymbol{I}} \delta(\boldsymbol{I}-\hat{\boldsymbol{I}}(s)) \\
& =-\nabla_{\boldsymbol{I}}\left[\left(-\mathbf{K}_{B} I+\boldsymbol{j}_{\boldsymbol{B}}\right) \delta(\boldsymbol{I}-\hat{\boldsymbol{I}}(s))\right],
\end{aligned}
$$

where $\mathbf{K}_{B}$ and $\boldsymbol{j}_{\boldsymbol{B}}$ are the absorption matrix and emission vector valid in the regime $\boldsymbol{B}, \nabla_{\boldsymbol{I}}$ is the nabla operator for the Stokes vector which is defined as

$\nabla_{\boldsymbol{I}}=\left(\begin{array}{c}\frac{\partial}{\partial I} \\ \frac{\partial}{\partial Q} \\ \frac{\partial}{\partial U} \\ \frac{\partial}{\partial V}\end{array}\right)$

Relation (24), allows us to write Eq. (23) as

$$
\begin{aligned}
\frac{\partial\langle\boldsymbol{I}\rangle}{\partial s}= & \iint-\nabla_{\boldsymbol{I}}\left[\left(-\mathbf{K}_{B} I+\boldsymbol{j}_{\boldsymbol{B}}\right) \delta(\boldsymbol{I}-\hat{\boldsymbol{I}}(s))\right] p(\boldsymbol{B}, s) \boldsymbol{I} \mathrm{d} \boldsymbol{I} \mathrm{d} \boldsymbol{B} \\
& +\iint \boldsymbol{I} \delta(\boldsymbol{I}-\hat{\boldsymbol{I}}(s)) \frac{\partial}{\partial s} p(\boldsymbol{B}, s) \mathrm{d} \boldsymbol{I} \mathrm{d} \boldsymbol{B} .
\end{aligned}
$$

If we now use the master Eq. (14) to express the spatial derivative of $p(\boldsymbol{B}, s)$, Eq. (26) can be written as,

$$
\begin{aligned}
\frac{\partial\langle\boldsymbol{I}\rangle}{\partial s}= & \iint-\nabla_{\boldsymbol{I}}\left[\left(-\mathbf{K}_{B} I+\boldsymbol{j}_{\boldsymbol{B}}\right) \delta(\boldsymbol{I}-\hat{\boldsymbol{I}}(s))\right] p(\boldsymbol{B}, s) \boldsymbol{I} \mathrm{d} \boldsymbol{I} \mathrm{d} \boldsymbol{B} \\
& +\iint\left(\int w\left(\boldsymbol{B}, \boldsymbol{B}^{\prime}\right) p\left(\boldsymbol{B}^{\prime}, s\right) \mathrm{d} \boldsymbol{B}^{\prime}\right) \boldsymbol{I} \delta(\boldsymbol{I}-\hat{\boldsymbol{I}}(s)) \mathrm{d} \boldsymbol{I} \mathrm{d} \boldsymbol{B} \\
& -\iint\left(\int w\left(\boldsymbol{B}^{\prime}, \boldsymbol{B}\right) p(\boldsymbol{B}, s) \mathrm{d} \boldsymbol{B}^{\prime}\right) \boldsymbol{I} \delta(\boldsymbol{I}-\hat{\boldsymbol{I}}(s)) \mathrm{d} \boldsymbol{I} \mathrm{d} \boldsymbol{B} .(27)
\end{aligned}
$$

Let us introduce the mean conditional Stokes vector (conditioned on the atmospheric regime $\boldsymbol{B}$ ) which is defined as

$\boldsymbol{Y}_{\boldsymbol{B}}(s)=\int \boldsymbol{I} p(\boldsymbol{I} \mid \boldsymbol{B}, s) \mathrm{d} \boldsymbol{I}$.

The components of the mean conditional Stokes vector are given in analogy to the definition of the regular Stokes vector,

$\boldsymbol{Y}_{B}=\left(\begin{array}{c}Y_{B}^{I} \\ Y_{B}^{Q} \\ Y_{B}^{U} \\ Y_{B}^{V}\end{array}\right)$

Taking into account Eq. (18) as well as the definition of the mean conditional Stokes vector we can integrate the first term on the r.h.s. of Eq. (27) by parts to obtain the following equation

$$
\begin{aligned}
& \frac{\partial}{\partial s} \int \boldsymbol{Y}_{B} p(\boldsymbol{B}, s) \mathrm{d} \boldsymbol{B}=\int\left(-\mathbf{K}_{B} Y_{B}+\boldsymbol{j}_{\boldsymbol{B}}\right) p(\boldsymbol{B}, s) \mathrm{d} \boldsymbol{B} \\
& +\int\left(\int w\left(\boldsymbol{B}, \boldsymbol{B}^{\prime}\right) \boldsymbol{Y}_{\boldsymbol{B}^{\prime}} p\left(\boldsymbol{B}^{\prime}, s\right) \mathrm{d} \boldsymbol{B}^{\prime}\right) \mathrm{d} \boldsymbol{B} \\
& -\int\left(\int w\left(\boldsymbol{B}^{\prime}, \boldsymbol{B}\right) \boldsymbol{Y}_{B} p(\boldsymbol{B}, s) \mathrm{d} \boldsymbol{B}^{\prime}\right) \mathrm{d} \boldsymbol{B},
\end{aligned}
$$

where we have made the reasonable assumption that the atmospheric vector $\boldsymbol{B}$ is independent from the Stokes intensities. If we now neglect the outer integration over the state space $\boldsymbol{B}$ and divide Eq. (30) by $p(\boldsymbol{B}, s)$ as well as taking into account the definition of the transition rate (A.2) we obtain the following integrodifferential equation for the mean conditional Stokes vector

$$
\begin{aligned}
\frac{\partial \boldsymbol{Y}_{B}}{\partial s}= & -\mathbf{K}_{B} Y_{B}+j_{\boldsymbol{B}}+\int \bar{\gamma}(\boldsymbol{B}) \boldsymbol{Y}_{\boldsymbol{B}^{\prime}} p\left(\boldsymbol{B}^{\prime}, s\right) \mathrm{d} \boldsymbol{B}^{\prime} \\
& -\int \gamma\left(\boldsymbol{B}^{\prime}\right) \boldsymbol{Y}_{B} p\left(\boldsymbol{B}^{\prime}\right) \mathrm{d} \boldsymbol{B}^{\prime} .
\end{aligned}
$$


Here we have introduced the modified fluctuation rate $\bar{\gamma}(\boldsymbol{B})$ which is given by

$\bar{\gamma}(\boldsymbol{B})=\frac{p(\boldsymbol{B})}{p(\boldsymbol{B}, s) \lambda(\boldsymbol{B})}$.

Equation (31) is the transport equation for the mean conditional Stokes vector $\boldsymbol{Y}_{B}$ which can be solved by specifying the initial conditions at the bottom of the atmosphere

$$
\begin{aligned}
\boldsymbol{Y}_{\boldsymbol{B}_{\mathbf{0}}}\left(\boldsymbol{s}_{\mathbf{0}}\right) & =\int \boldsymbol{I}_{0} p\left(\boldsymbol{I}_{0} \mid \boldsymbol{B}_{0}, s_{0}\right) \mathrm{d} \boldsymbol{I}_{0} \\
& =\int \boldsymbol{I}_{0} \delta\left(\boldsymbol{I}_{0}-\boldsymbol{I}\left(s_{0}\right)\right) \mathrm{d} \boldsymbol{I}_{0},
\end{aligned}
$$

as well as an initial probability distribution $p\left(\boldsymbol{B}_{0}, s_{0}\right)$. Equation (31) can be simplified under the assumption that the fluctuation rate $\gamma$ does not depend on the particular magnetic regime which then allows us to write

$\frac{\partial \boldsymbol{Y}_{B}}{\partial s}=\int-\mathbf{K} Y_{B}+\boldsymbol{j}+\bar{\gamma}\langle\boldsymbol{I}(s)\rangle-\gamma \boldsymbol{Y}_{B}$

The mean unconditional Stokes vector at the top of the atmosphere $s_{t}$ - our observable quantity - can then be readily obtained by a final integration of the mean conditional Stokes vector over the entire state space of $\boldsymbol{B}$,

$\left\langle\boldsymbol{I}\left(s_{t}\right)\right\rangle=\int \boldsymbol{Y}_{\boldsymbol{B}}\left(s_{t}\right) p\left(\boldsymbol{B}, s_{t}\right) \mathrm{d} \boldsymbol{B}$.

Equation (31) describes the evolution of the mean conditional Stokes vector through the atmosphere from which the mean Stokes vector, the macroscopic (observable) quantity can be obtained. The stochastic transport equation for the mean conditional Stokes vector (31) has the general form of a stochastic master equation (van Kampen 1992) and describes the transport of photons which are subject to the familiar processes of absorption (thermalization) and emission as well as two processes which describes the flow (fluctuation) between the different atmospheric regimes or components of the atmosphere. To understand the two new terms in the stochastic transport equation it is important to realize that Eq. (31) is conditioned on one particular atmospheric regime $\boldsymbol{B}$ such that the integration process is entirely performed within that particular regime. Hence ,the two new terms in the stochastic transfer equation statistically couples the conditional transport equation for $\boldsymbol{B}$ to the other atmospheric regimes. In detail, the third term on the r.h.s. of Eq. (31) gives the amount of intensity or photons which enters per unit length scale from all other atmospheric regimes into the regime $\boldsymbol{B}$ under consideration, this term therefore describes an additional statistical inflow or source term, while the fourth term describes the loss of intensity per unit length scale due to transitions of photons to other possible regimes and therefore adds an additional statistical absorption. Due to the resemblance to the transport equation in a scattering medium both statistical terms can be considered as statistical scattering terms which describe the scattering of photons in and from the regime $\boldsymbol{B}$. The stochastic transfer Eq. (31) can be written in a more compact form if we combine the statistical source term with the emission vector $\boldsymbol{j}_{\boldsymbol{B}}$ and the statistical absorption with the absorption matrix $\mathbf{K}_{B}$. We can then define a modified (conditional) absorption matrix $\tilde{\mathbf{K}}_{B}$ such as

$\tilde{\mathbf{K}}_{B}=\mathbf{K}_{B}+\mathbf{1} \gamma$,

where $\mathbf{1}$ is the unit $4 \times 4$ matrix. The modified (conditional) emission vector $\tilde{\boldsymbol{j}}$ can be defined as

$\tilde{\boldsymbol{j}}_{\boldsymbol{B}}=\boldsymbol{j}_{\boldsymbol{B}}+\int \bar{\gamma}(\boldsymbol{B}) \boldsymbol{Y}_{\boldsymbol{B}^{\prime}} p\left(\boldsymbol{B}^{\prime}, s\right) \mathrm{d} \boldsymbol{B}^{\prime}$.
This allows us to write the stochastic transport Eq. (31) in the following form

$\frac{\partial \boldsymbol{Y}_{B}}{\partial s}=-\tilde{\mathbf{K}}_{B} Y_{B}+\tilde{\boldsymbol{j}}_{\boldsymbol{B}}$

In the case of a discrete state space $\boldsymbol{B}$ we can obtain the discrete form of the stochastic transport Eq. (31) from the discrete master equation Eq. (A.10) which gives

$$
\begin{aligned}
\frac{\partial \boldsymbol{Y}_{\boldsymbol{B}_{m}}}{\partial s}= & -\mathbf{K}_{B} Y_{B_{m}}+\boldsymbol{j}_{\boldsymbol{B}}+\sum_{n} \bar{\gamma}(\boldsymbol{B}) \boldsymbol{Y}_{\boldsymbol{B}_{n}} p\left(\boldsymbol{B}_{\boldsymbol{n}}, s\right) \\
& -\sum_{n} \gamma\left(\boldsymbol{B}^{\prime}\right) \boldsymbol{Y}_{\boldsymbol{B}_{m}} p\left(\boldsymbol{B}_{\boldsymbol{n}}, s\right) .
\end{aligned}
$$

\subsection{The formal solution}

A formal solution for the mean conditional Stokes vector can be obtained in analogy to the formal solution of the polarized radiative transfer equation (Landi Degl'Innocenti \& Landi Degl'Innocenti 1985). Taking into account the definition of the modified absorption matrix (36) the transfer Eq. (38) in a purely absorbing medium can be written as

$$
\frac{\partial \boldsymbol{Y}_{B}}{\partial s}=-\tilde{\mathbf{K}}_{B} \boldsymbol{Y}_{B}
$$

Since the statistical absorption is a symmetric operation and does not depend on the polarization state of the mean conditional Stokes vector we can introduce a modified conditional matrix attenuation or evolution operator which acts on the mean conditional Stokes vector $\tilde{\boldsymbol{O}}_{B}\left(s, s^{\prime}\right)$ at the position $s^{\prime}$ to give the mean conditional Stokes vector at the position $s$

$\boldsymbol{Y}_{B}(s)=\tilde{\boldsymbol{O}}_{B}\left(s, s^{\prime}\right) \boldsymbol{Y}_{\boldsymbol{B}}\left(s^{\prime}\right)$

and obeys the following limiting condition

$\tilde{\boldsymbol{O}}_{B}(s, s)=1$.

Taking the derivative of (40) and taking into account the relation (41) a differential equation for the conditional evolution operator can be written as

$\frac{\partial \tilde{\boldsymbol{O}}_{B}\left(s, s^{\prime}\right)}{\partial s}=\tilde{\mathbf{K}}_{B}(s) \tilde{\boldsymbol{O}}_{B}\left(s, s^{\prime}\right)$

Following Landi Degl'Innocenti \& Landi Degl'Innocenti (1985), we can readily obtain the formal solution for the inhomogeneous case (31) by a direct substitution, such that we can write

$\boldsymbol{Y}_{\boldsymbol{B}}(s)=\int_{s_{0}}^{s} \tilde{\boldsymbol{O}}_{B}\left(s, s^{\prime}\right) \tilde{\boldsymbol{j}}_{\boldsymbol{B}}\left(s^{\prime}\right) \mathrm{d} s^{\prime}+\tilde{\boldsymbol{O}}_{B}\left(s, s_{0}\right) \boldsymbol{Y}_{\boldsymbol{B}}\left(s_{0}\right)$

where $\tilde{\boldsymbol{j}}_{\boldsymbol{B}}\left(s^{\prime}\right)$ is the modified conditional emission vector (37). In the special case of a constant modified absorption matrix $\tilde{\mathbf{K}}_{B}$ the conditional evolution operator can be written as

$\tilde{\boldsymbol{O}}_{B}\left(s, s^{\prime}\right)=\exp \left[-\left(s-s^{\prime}\right) \tilde{\mathbf{K}}_{B}\right]$,

where the exponential of the modified absorption matrix is given by its Taylor expansion. Thus we see that by introducing a modified conditional evolution operator the formal solution for the stochastic polarized radiative transfer Eq. (31) is formally identical to the deterministic case (Landi Degl'Innocenti \& Landi Degl'Innocenti 1985). 


\subsection{Micro- and macroturbulent limits}

In this section we show that the stochastic transfer Eq. (31) contains the microturbulent as well as the macroturbulent approach as limiting cases.

We begin by considering the macroturbulent case which follows the idea that the turbulence or the stochastic character of the atmosphere is exclusively perpendicular to the line-of-sight. Any fluctuation along the line-of-sight is by far larger than the mean free path of the photons and is therefore negligible. On the other hand, the atmosphere is highly structured in the plane perpendicular to the line-of-sight. For observations with finite resolution, there always exists an ensemble of individual line-ofsights which traversing through different atmospheric regimes. The statistical variation within that finite resolution element is usually described by a simple probability density function. Macroturbulence, whether for velocity field, magnetic fields or a more general atmospheric vector like $\boldsymbol{B}$, certainly provides only a crude and rather simplistic representation of a turbulent or fluctuating atmosphere with large coherent structures. In terms of our mesostructured approach, macroturbulence can be described by a fluctuation rate that goes to zero or a correlation length that goes to infinity respectively. If we therefore consider Eq. (31) and take the limit as $\gamma \rightarrow 0$ or $\lambda \rightarrow \infty$ respectively, we obtain the transport equation for the mean conditional Stokes vector $\boldsymbol{Y}_{B}$ in the macrostructured or macroturbulent limit

$\lim _{\lambda \rightarrow \infty} \frac{\partial \boldsymbol{Y}_{B}}{\partial s}=-\mathbf{K}_{B} Y_{B}+\boldsymbol{j}_{\boldsymbol{B}}$.

This leads to a deterministic transport equation where both statistical scattering terms vanish and the conditional Stokes vector in the regime $\boldsymbol{B}$ completely decouples from all other atmospheric components. A final integration over the state space at the top of the atmosphere (Eq. (35)) gives the mean observable Stokes vector for each frequency

$\langle\boldsymbol{I}(s)\rangle=\int \boldsymbol{Y}_{\boldsymbol{B}}(s) p(\boldsymbol{B}, s) \mathrm{d} \boldsymbol{B}$.

In the case of a random macroscopic velocity field where $\boldsymbol{B}=\boldsymbol{v}$ and a Gaussian LOS velocity distribution $\mathrm{p}(\mathrm{v})$ this equation turns into the familiar (macroturbulent) convolution integral.

To derive the microturbulent limit, we have to take into account that the transport Eq. (31) was derived in the particular limit of a small ratio of the path element $\Delta s$ to the correlation length $\lambda$ (see Appendix A) and therefore the limit in Eq. (31) cannot be performed directly. Instead, let us first consider the underlying Kubo-Anderson process Eq. (12) where we take the limit as $\gamma \rightarrow \infty$ or $\lambda \rightarrow 0$,

$\lim _{\lambda \rightarrow 0} p\left(\boldsymbol{B}^{\prime \prime}, s+\Delta s \mid \boldsymbol{B}^{\prime}, s\right)=p\left(\boldsymbol{B}^{\prime \prime}\right)$.

The transition probability from $\boldsymbol{B}^{\prime}$ to $\boldsymbol{B}^{\prime \prime}$ is then exclusively determined by the probability of final state $p\left(\boldsymbol{B}^{\prime \prime}\right)$ and, hence, entirely uncorrelated from the initial state $\boldsymbol{B}^{\prime}$. The Kubo-Anderson process for this limit is therefore simply given by the overall stationary (s-independent) probability density function $p(\boldsymbol{B})$ of the atmosphere. Since the Kubo-Anderson process is now independent of the spatial variable $s$ it follows that the master Eq. (14) is stationary as well and must be identical to zero. As the conditional Stokes vectors are in general $\boldsymbol{Y}_{\boldsymbol{B}} \neq 0$ this further implies that the sum of the second and third term on the r.h.s. of Eq. (31) must be in an equilibrium state such that both will mutually cancel. This equilibrium can be expressed by the following relation,

$\bar{\gamma} \int \boldsymbol{Y}_{\boldsymbol{B}^{\prime}} p\left(\boldsymbol{B}^{\prime}\right) \mathrm{d} \boldsymbol{B}^{\prime}=\gamma \int \boldsymbol{Y}_{B} p\left(\boldsymbol{B}^{\prime}\right) \mathrm{d} \boldsymbol{B}^{\prime}$
From Eqs. (48) and (32), we see that $\bar{\gamma}=\gamma$ and Eq. (49) can be written as

$\gamma \int\left(\boldsymbol{Y}_{\boldsymbol{B}^{\prime}}-\boldsymbol{Y}_{B}\right) p\left(\boldsymbol{B}^{\prime}\right) \mathrm{d} \boldsymbol{B}^{\prime}=0$.

From this relation it immediately follows that

$\boldsymbol{Y}_{B}=\langle\boldsymbol{I}\rangle$.

This relation is a direct consequence of the strong statistical coupling between the conditional Stokes vectors. If we insert the relation (51) into the transport Eq. (31) and taking into account relation (49) we can write the transport equation for the mean conditional Stokes vector in the microturbulent limit as

$\lim _{\lambda \rightarrow \infty} \frac{\partial \boldsymbol{Y}_{B}}{\partial s}=-\mathbf{K}_{B}\langle\boldsymbol{I}\rangle+\boldsymbol{j}_{\boldsymbol{B}}$

Note that a strong statistical coupling of the conditional Stokes vectors, expressed by a small correlation length, leads to a rapid fluctuation of the atmospheric conditions along the photon trajectory. Due to this strong coupling and high transition probabilities, photons will fluctuate on very small scales many times from one atmospheric regime to another before they eventually get thermalized or escape from the atmosphere. The high probability for a statistical absorption and scattering event compared to the thermalization probability provides an almost complete mixing on smallest scales such that the conditional Stokes vector will rapidly converge to the mean Stokes vector (see relation (51)). The microturbulent transport equation for the mean unconditional Stokes vector can then be derived from Eq. (35) to give

$\frac{\partial\langle\boldsymbol{I}\rangle}{\partial s}=-\int \mathbf{K}_{B}\langle\boldsymbol{I}\rangle p(\boldsymbol{B}) \mathrm{d} \boldsymbol{B}+\int \boldsymbol{j}_{\boldsymbol{B}} p(\boldsymbol{B}) \mathrm{d} \boldsymbol{B}$

using $\langle I\rangle \cong I$, we can write the transport equation for the mean Stokes vector in the microturbulent limit finally as

$\frac{\partial \boldsymbol{I}}{\partial s}=\langle\mathbf{K}\rangle \boldsymbol{I}+\langle\boldsymbol{j}\rangle$

Note that, mathematically, the microturbulent limit is reached in the strict limit of $\lambda \rightarrow 0$ or $\gamma \rightarrow \infty$. However, as the complete mixing of the photons or the ratio of statistical scattering to true absorption is in fact controlled by the opacities in the individual atmospheric regimes the effective microturbulent limit will be reached for a finite correlation length (see Sect. 4).

\subsection{Stokes profile asymmetries}

The asymmetry properties of Stokes profiles originating in a stratified atmosphere were the subject of several studies over the recent decades (Auer \& Heasley 1978; Landi Degl'Innocenti \& Landolfi 1983; Sanchez Almeida et al. 1989; Solanki \& Montavon 1993; Grossmann-Doerth et al. 2000; Steiner 2000; López Ariste 2002). In the following, we show how the stochastic transfer equation for polarized light can be used to describe the generation of asymmetric Stokes profiles. We assume that the atmosphere comprises an ensemble of different magnetic regimes where each regime may have its own thermal properties and different LOS velocities. For simplicity, we assume that each regime or component is height-independent such that each conditional absorption matrix $\mathbf{K}_{B}$ is constant with $s$. Note again, that the vector $\boldsymbol{B}$ contains all relevant atmospheric parameters. According to Landi Degl'Innocenti \& Landi Degl'Innocenti (1981) for the Zeeman effect in an isolated triplet the seven 
entries of the absorption matrix possess clear symmetry properties with respect to the central line frequency or wavelength at any given depth. The point of symmetry of the absorption profiles in the absorption matrix are defined with respect to the central frequency $v_{0}$ of the underlying Voigt- or Faraday-Voigt profiles. If we denote $v$ as the distance from the the central frequency $v_{0}$, then the entries of the absorption matrix $\mathbf{K}_{v_{0}}$ satisfy the following relations

$$
\begin{aligned}
& \eta_{I}(v)=\eta_{I}(-v) \\
& \eta_{Q}(v)=\eta_{Q}(-v) \\
& \eta_{U}(v)=\eta_{U}(-v) \\
& \eta_{V}(v)=-\eta_{V}(-v) \\
& \rho_{Q}(v)=-\rho_{Q}(-v) \\
& \rho_{U}(v)=-\rho_{U}(-v) \\
& \rho_{V}(v)=\rho_{V}(-v) .
\end{aligned}
$$

This is a direct consequence of the symmetry properties of the Voigt and Faraday-Voigt function. The absorption profiles $\eta_{I}, \eta_{Q}, \eta_{U}, \rho_{V}$ are even functions regarding to the central frequency $v_{0}$ and the $\eta_{V}, \rho_{Q}, \rho_{U}$, are odd functions. We recall that the evolution operator in the case of a constant absorption matrix K (Landi Degl'Innocenti \& Landi Degl'Innocenti 1985) can be written as

$\boldsymbol{O}\left(s, s^{\prime}\right)=\exp ^{\left[-\mathbf{K}\left(s-s^{\prime}\right)\right]}=\sum_{n=0}^{\infty} \frac{-\left[\mathbf{K}\left(s-s^{\prime}\right)\right]^{n}}{n !}$.

Note that the evolution operator itself shares the same symmetry properties about the central frequency $v_{0}$ as the entries of the absorption matrix $\mathbf{K}$. We can now state, that any four component vector $\boldsymbol{X}$ whose components shares the following symmetry properties in the frequency domain

$$
\begin{aligned}
& X_{v_{0}}^{1} \propto \eta_{I v_{0}} \\
& X_{v_{0}}^{2} \propto \eta_{Q v_{0}} \\
& X_{v_{0}}^{3} \propto \eta_{U v_{0}} \\
& X_{v_{0}}^{4} \propto \eta_{V v_{0}},
\end{aligned}
$$

and which is subject to a transformation by means of the evolution operator (56) will preserve the symmetry properties. This can be verified by a direct multiplication, and is a direct consequence of the symmetry behavior (the even-odd relation) of the individual absorption profiles in the absorption matrix. Therefore, any evolution operator (56) that acts on a vector $\boldsymbol{X}$ with the properties (57), and which shares the same central symmetry $v_{0}$ leaves the symmetry properties of the vector components unaltered. On the other hand, non-symmetric entries or entries with different points of symmetry in the evolution operator matrix will in general not preserve the symmetry of the vector $\boldsymbol{X}$.

For the sake of clarity we change the notation from a continuous state space to a discrete, such that the individual atmospheric components $\boldsymbol{B}_{k}$ can be better distinguished. If we now take the formal solution for the mean conditional Stokes vector $\boldsymbol{Y}_{B_{k}}$ in a semi-infinite atmosphere we get

$\boldsymbol{Y}_{B_{k}}(s)=\int_{-\infty}^{s} \tilde{\boldsymbol{O}}_{B_{k}}\left(s, s^{\prime}\right) \tilde{\boldsymbol{j}}_{B_{k}}\left(s^{\prime}\right) \mathrm{d} s^{\prime}$.

We then use the explicit form of the modified emission $\tilde{\boldsymbol{j}}_{B_{k}}\left(s^{\prime}\right)$, which is the sum of the thermal emission $\boldsymbol{j}_{B}^{\text {Therm }}$ and the inflow from the statistical scattering $\boldsymbol{j}_{B}^{\text {Scat }}$ we can write Eq. (58) in the form

$$
\begin{aligned}
\tilde{\boldsymbol{j}}_{B_{k}}(s) & =\boldsymbol{j}_{B_{k}}^{\text {Therm }}(s)+\boldsymbol{j}_{B_{k}}^{\text {Scat }}(s) \\
& =\boldsymbol{j}_{B_{k}}^{\text {Therm }}(s)+\sum_{l} \bar{\gamma}\left(\boldsymbol{B}_{k}, s\right) \boldsymbol{Y}_{\boldsymbol{B}_{l}} p\left(\boldsymbol{B}_{l}, s\right) .
\end{aligned}
$$

From the symmetry properties of the evolution operator $\tilde{\boldsymbol{O}}_{B_{k}}\left(s, s^{\prime}\right)$ and the thermal emission vector $\boldsymbol{j}_{B_{k}}^{\text {Therm }}$ (note, both acting in the same regime $\boldsymbol{B}_{k}$ ) it is clear that a transformation of $\boldsymbol{j}_{B_{k}}^{\text {Therm }}$ by the evolution operator $\tilde{\boldsymbol{O}}_{B_{k}}\left(s, s^{\prime}\right)$ leaves the symmetry properties of $\boldsymbol{j}_{B_{k}}^{\text {Therm }}$ unaltered and therefore does not lead to any asymmetry of the conditional Stokes vector. This simply reflects the fact that we have assumed that the absorption matrix for each atmospheric component is constant. We now concentrate on the statistical scattering term and replace $\boldsymbol{Y}_{\boldsymbol{B}_{l}}$ on the r.h.s. of Eq. (59) by its formal solution and then insert the expression for the statistical scattering into Eq. (58) which gives

$$
\begin{aligned}
\boldsymbol{Y}_{B_{k}}(s)= & \int_{-\infty}^{s} \int_{-\infty}^{s^{\prime}} \tilde{\boldsymbol{O}}_{B_{k}}\left(s, s^{\prime}\right) \sum_{l} \tilde{\boldsymbol{O}}_{\boldsymbol{B}_{l}}\left(s^{\prime}, s^{\prime \prime}\right) \\
& \times \tilde{\boldsymbol{j}}_{\boldsymbol{B}_{l}}\left(s^{\prime \prime}\right) \bar{\gamma}\left(\boldsymbol{B}_{k}, s^{\prime}\right) p\left(\boldsymbol{B}_{l}, s^{\prime \prime}\right) \mathrm{d} s^{\prime \prime} \mathrm{d} s^{\prime} .
\end{aligned}
$$

Equation (60) leads to a sum of successive applications of the local evolution operators $\tilde{\boldsymbol{O}}_{B_{k}}$ and $\tilde{\boldsymbol{O}}_{\boldsymbol{B}_{l}}$. Despite the fact that each atmospheric component $\boldsymbol{B}_{l}$ has a constant absorption matrix, the individual atmospheric components differ in their atmospheric parameters such that the matrix exponentials (evolution operators) in general do not commute. We therefore use the Baker-Campbell-Hausdorff formula (Magnus 1954) to express the product of two matrix exponential which involves all higherorder terms of the commutator, $[\boldsymbol{A}, \boldsymbol{B}]=\boldsymbol{A} \boldsymbol{B}-\boldsymbol{B} \boldsymbol{A}$,

$$
\begin{aligned}
\exp (\boldsymbol{A}) \exp (\boldsymbol{B})= & \exp \left(\boldsymbol{A}+\boldsymbol{B}+\frac{1}{2}[\boldsymbol{A}, \boldsymbol{B}]\right. \\
& \left.+\frac{1}{12}[A,[\boldsymbol{A}, \boldsymbol{B}]]-\frac{1}{12}[B,[\boldsymbol{A}, \boldsymbol{B}]]+\ldots\right) .
\end{aligned}
$$

Using this relation we can write Eq. (60) as

$$
\begin{aligned}
\boldsymbol{Y}_{B_{k}}(s)= & \int_{-\infty}^{s} \int_{-\infty}^{s^{\prime}} \sum_{l} \exp \left(\mathbf{K}_{B_{k}}\left(s^{\prime}-s\right)+\mathbf{K}_{B_{l}}\left(s^{\prime \prime}-s^{\prime}\right)\right. \\
& \left.+\frac{1}{2}\left[\mathbf{K}_{B_{k}}, \mathbf{K}_{B_{l}}\right]\left(s^{\prime}-s\right)\left(s^{\prime \prime}-s^{\prime}\right)+\ldots\right) \\
& \times \tilde{\boldsymbol{j}}_{\boldsymbol{B}_{l}}\left(s^{\prime \prime}\right) \bar{\gamma}\left(\boldsymbol{B}_{k}, s^{\prime}\right) p\left(\boldsymbol{B}_{l}, s^{\prime \prime}\right) \mathrm{d} s^{\prime \prime} \mathrm{d} s^{\prime}
\end{aligned}
$$

Although each individual absorption matrix $\mathbf{K}_{B_{l}}$ has its own central frequency $v_{l}$ (point of symmetry) due to the individual atmospheric conditions, the superposition and multiplication of the individual matrices will in general not retain or lead to a new symmetry in the compound matrix and therefore the resulting (combined) evolution operator will not possess any symmetry properties. This means that the modified emission vector $\tilde{\boldsymbol{j}}_{\boldsymbol{B}_{l}}\left(s^{\prime \prime}\right)$ in Eq. (62), which again receives contributions from thermal emission and statistical scattering, is subject to a non-symmetric compound evolution operator of the form

$$
\begin{aligned}
\tilde{\boldsymbol{O}}_{B_{k l}}\left(s, s^{\prime}\right)= & \sum_{l} \exp \left(\mathbf{K}_{B_{k}}\left(s^{\prime}-s\right)+\mathbf{K}_{B_{l}}\left(s^{\prime \prime}-s^{\prime}\right)\right. \\
& \left.+\frac{1}{2}\left[\mathbf{K}_{B_{k}}, \mathbf{K}_{B_{l}}\right]\left(s^{\prime}-s\right)\left(s^{\prime \prime}-s^{\prime}\right)+\ldots\right) .
\end{aligned}
$$

Regardless, of the symmetry properties of the statistical scattering vector $j_{\boldsymbol{B}_{l}}^{\text {Scat }}\left(s^{\prime \prime}\right)$ at $s^{\prime \prime}$ - which itself is the result of the underlying mixed atmosphere - the symmetry of the thermal emission 
vector $\boldsymbol{j}_{\boldsymbol{B}_{l}}^{\text {Therm }}\left(s^{\prime \prime}\right)$, which shares the local symmetry properties of the regime $\boldsymbol{B}_{l}$, is no longer preserved by a transformation with the compound evolution operator (63) and thus no general symmetry property for the conditional Stokes vector $\boldsymbol{Y}_{B_{k}}$ can evolve in this type of stochastic atmosphere. So we can conclude that it is the inherent asymmetry of the compound evolution operator (63) that prevents any propagation of symmetric profiles.

Yet another interesting fact that directly follows from Eq. (62) is that the degree of asymmetry is proportional to the fluctuation rate $\gamma\left(\boldsymbol{B}_{k}\right)$ or inversely proportional to the correlation length $\lambda\left(\boldsymbol{B}_{k}\right)$. This fact will be used in the following section to introduce the correlation length as a diagnostic parameter.

\section{Numerical experiments}

In this section we perform a number of numerical experiments to demonstrate how mesostructures, structures of finite length, can affect the polarized line formation and the shape of Stokes profiles. Particular emphasis in this section is given to the generation of a asymmetric Stokes profiles and their dependence on the mean length scales of the underlying magnetic and non-magnetic structures. We show, how a wide range of structural length scales are not appropriately described by a microturbulent or macroturbulent approach and that significant deviations occur compared to our more realistic and accurate mesostructured approach.

\subsection{Numerical solution}

Before we begin with our model calculation we give a brief outline of the numerical procedure we have used to solve the stochastic transport equations. The numerical solution is based on the discrete form of transport equation for the mean conditional Stokes vector (39) which is given as

$$
\begin{aligned}
\frac{\partial \boldsymbol{Y}_{\boldsymbol{B}_{m}}}{\partial s}= & -\mathbf{K}_{B} Y_{B_{m}}+\boldsymbol{j}_{\boldsymbol{B}}+\sum_{n} \bar{\gamma}(\boldsymbol{B}) \boldsymbol{Y}_{\boldsymbol{B}_{n}} p\left(\boldsymbol{B}_{\boldsymbol{n}}, s\right) \\
& -\sum_{n} \gamma\left(\boldsymbol{B}^{\prime}\right) \boldsymbol{Y}_{\boldsymbol{B}_{\boldsymbol{m}}} p\left(\boldsymbol{B}_{\boldsymbol{n}}, s\right) .
\end{aligned}
$$

Each of the transport equations for the mean conditional Stokes vector couples the 4 mean conditional Stokes parameter defined in Eq. (29) via the absorption matrix. Additionally, the transport equations are coupled via the statistical absorption and scattering terms to the $n$ different atmospheric regimes. We therefore express the whole system by means of a $4 n \times 4 n$ system matrix $\mathbf{M}$ which gives us the following system of differential equations

$$
\frac{\mathrm{d} \hat{\boldsymbol{Y}}}{\mathrm{d} s}=\mathbf{M} \hat{\boldsymbol{Y}}+\hat{\boldsymbol{j}}
$$

where $\hat{\boldsymbol{Y}}$ and $\hat{\boldsymbol{j}}$ are the $4 n$-element Stokes vector and thermal emission vector. The system matrix $\mathbf{M}$ can be compactly written by introducing the local submatrices $\mathbf{R}_{i}$ and $\mathbf{S}_{i}$ to yield the following system of differential equations

$\frac{\mathrm{d}}{\mathrm{d} s}\left(\begin{array}{c}Y_{B_{1}}^{I}(s) \\ Y_{B_{1}}^{Q}(s) \\ Y_{B_{1}}^{U}(s) \\ Y_{B_{1}}^{V}(s) \\ Y_{B_{2}}^{I}(s) \\ Y_{B_{2}}^{Q}(s) \\ Y_{B_{2}}^{U}(s) \\ Y_{B_{2}}^{V}(s) \\ \cdot \\ \cdot \\ \cdot \\ Y_{B_{n}}^{I}(s) \\ Y_{B_{n}}^{Q}(s) \\ Y_{B_{n}}^{U}(s) \\ Y_{B_{n}}^{V}(s)\end{array}\right)=\left(\begin{array}{ccccc}\mathbf{R}_{1} & \mathbf{S}_{2} & \mathbf{S}_{3} & \cdots & \mathbf{S}_{n} \\ \mathbf{S}_{1} & \mathbf{R}_{2} & \mathbf{S}_{3} & \cdots & \mathbf{S}_{n} \\ \mathbf{S}_{1} & \mathbf{S}_{2} & \mathbf{R}_{3} & \cdots & \mathbf{S}_{n} \\ \cdot & \cdot & \cdot & & \cdot \\ \cdot & \cdot & \cdot & & \cdot \\ \cdot & \cdot & \cdot & & \cdot \\ \mathbf{S}_{1} & \mathbf{S}_{2} & \mathbf{S}_{3} & \cdots & \mathbf{R}_{n}\end{array}\right]\left(\begin{array}{c}Y_{B_{1}}^{I} \\ Y_{B_{1}}^{Q} \\ Y_{B_{1}}^{U} \\ Y_{B_{1}}^{V} \\ Y_{B_{2}}^{I} \\ Y_{B_{2}}^{Q} \\ Y_{B_{2}}^{U} \\ Y_{B_{2}}^{V} \\ \cdot \\ \cdot \\ \cdot \\ \\ \end{array}\right.$

The local absorption matrix $\mathbf{R}_{i}$ of the regime $i$ is given by the following $4 \times 4$ matrix

$\mathbf{R}_{i}=\left[\begin{array}{cccc}-\left(\eta_{B_{i}}^{I}+\frac{1}{\lambda\left(B_{i}\right)}\right) & -\eta_{B_{i}}^{Q} & -\eta_{B_{i}}^{U} & -\eta_{B_{i}}^{V} \\ -\eta_{B_{i}}^{Q} & -\left(\eta_{B_{i}}^{I}+\frac{1}{\lambda\left(B_{i}\right)}\right) & -\rho_{B_{i}}^{V} & \rho_{B_{i}}^{U} \\ -\eta_{B_{i}}^{U} & \rho_{B_{i}}^{V} & -\left(\eta_{B_{i}}^{I}+\frac{1}{\lambda\left(B_{i}\right)}\right) & -\rho_{B_{i}}^{Q} \\ -\eta_{B_{i}}^{V} & -\rho_{B_{i}}^{U} & \eta_{B_{i}}^{Q} & -\left(\eta_{B_{i}}^{I}+\frac{1}{\lambda\left(B_{i}\right)}\right)\end{array}\right]$.

And the local statistical scattering matrix $\mathbf{S}_{i}$ is given by

$\mathbf{S}_{i}=\left[\begin{array}{cccc}-\frac{p\left(B_{i}\right)}{\lambda_{B_{i}}} & 0 & 0 & 0 \\ 0 & -\frac{p\left(B_{i}\right)}{\lambda_{B_{i}}} & 0 & 0 \\ 0 & 0 & -\frac{p\left(B_{i}\right)}{\lambda_{B_{i}}} & 0 \\ 0 & 0 & 0 & -\frac{p\left(B_{i}\right)}{\lambda_{B_{i}}}\end{array}\right]$

The system is integrated by a fourth-order Runge-Kutta method (Press et al. 1992) with appropriate boundary values for the mean conditional vectors $\boldsymbol{Y}_{\boldsymbol{B}_{m}}$. The initial conditions are given according to Eq. (33).

$\boldsymbol{Y}_{\boldsymbol{B}_{\boldsymbol{m}}}=S_{\mathrm{c}}\left(\tau_{i_{N}}\right) \boldsymbol{e}_{0}+\left[\frac{\mathrm{d} S_{\mathrm{c}}\left(\tau_{i_{N}}\right)}{\mathrm{d} s}\right] \mathbf{K}^{-1}\left(\tau_{i_{N}}\right) \boldsymbol{e}_{0}$,

where $\boldsymbol{e}_{0}$ is the unity vector and $S_{\mathrm{c}}$ is the Planck function in the LTE case. The lower boundaries are chosen for a sufficient large optical depth such that each of the different atmospheric regimes satisfy the condition $\tau_{i_{N}} \geq 2$.

To obtain the mean Stokes vector at the top of the atmosphere we finally sum up the individual contributions of the mean conditional Stokes vectors according to Eq. (35).

\subsection{A mesostructured magnetic atmosphere -2 fluctuating components}

To demonstrate the importance of the correlation length for the line formation process we begin by considering a simple model of a stochastic atmosphere consisting of two different types of components.

The arrangement of the different structures is such that we assume to have an ensemble of magnetic structures which are embedded in field-free regions. This picture is very similar to the often invoked two-component flux tube scenario often used for modeling unresolved magnetic structures in the solar network 
and internetwork. However, this scenario differs twofold, first, the resolution element comprises a whole ensemble of many individual structures and is not limited to a single static flux tube with a prescribed geometry, and second, the length scale of the individual magnetic and non-magnetic structures are finite. In the following we prefer therefore the term ensemble rather than components to emphasize the intrinsic complexity of the atmosphere. The line-of-sight length scale of the individual magnetic and non-magnetic structures in our model will be determined by the same correlation length $L$.

The individual magnetic structures in our model share the same physical parameters, the same holds for the non-magnetic structures. Note also, that we make no particular assumptions about the geometry of the magnetic flux structures and the ambient non-magnetic velocity field. For simplicity reasons we assume the same temperature stratification for the magnetic and the field-free structures. This should be a reasonable choice for small scale magnetic structures $(<100 \mathrm{~km}$ in horizontal diameter) where thermal conduction by radiation efficiently smears out temperature differences. But even for larger structures it has no significant influence on the qualitative behavior of the here presented model calculations. The underlying temperature and pressure structure is that of the quiet sun model atmosphere, HOLMUL, of Holweger \& Mueller (1974). The magnetic field strength within the magnetic structures is assumed to be $500 \mathrm{G}$ (Gauss) with no internal LOS velocity. In the ambient medium we assume a downflow LOS velocity of $550 \mathrm{~m} / \mathrm{s}$ to mimic a intergranular downflow. The total filling factor of the magnetic structures, which corresponds to the probability value in our stochastic scenario, is set to 5\%. Despite its simplicity this model should reflect the essential features of magnetic flux structures embedded in intergranular lanes. For this numerical experiment we have used the iron line Fe I $26302 \AA$. The coupled discrete stochastic transport Eq. (39) for each conditional Stokes vector is solved according to the numerical procedure described in Sect. (4.1). Note again, that we use the same correlation length $L$ for both ensemble structures such that $L$ is independent of the particular atmospheric regime.

We now begin to change the correlation length $L$ from very small (microturbulent) to very large values (macroturbulent) which results in a gradual increase in the (line-of-sight) order of the system (atmosphere). Such a decrease of the fluctuation rate should also affect the Stokes $V$ profile asymmetry in characteristic way, because of the distinct dependence of the asymmetry on the correlation length $L$ (see Sect. 3.4). To quantify the degree of Stokes $V$ amplitude and area asymmetry we adopt the usual definition for the amplitude asymmetry $\delta a$,

$\delta a=\frac{\left|a_{b}\right|-\left|a_{r}\right|}{\left|a_{b}\right|+\left|a_{r}\right|}$

and the area asymmetry $\delta A$,

$\delta A=s_{b} \frac{\int_{\Lambda} V \mathrm{~d} \lambda}{\int_{\Lambda}|V| \mathrm{d} \lambda}$,

where $a_{b}$ and $a_{r}$ are the extrema of the blue and red lobes of the Stokes $V$ signal and $s_{b}$ the sign of the blue lobe. The integration for the area asymmetry $\delta A$ is performed over the entire wavelength range $\Lambda$ of the considered Stokes $V$ signal.

Figure 1 now shows how the amplitude and area asymmetry of the Stokes $V$ profile varies with the correlation length $L$ of the magnetic and non-magnetic structures. For the broad range of meso-scales $(20-1000 \mathrm{~km})$ we see the expected rapid decrease of the asymmetries with increasing correlation length $L$.
This rapid decay already starts at a correlation length of approximately $15 \mathrm{~km}$. For very small correlation lengths, the asymmetries (amplitude and area) saturate and begin to converge into the microturbulent limit. For a better comparison we have also calculated the expected amplitude and area asymmetries in the microturbulent limit, where we have used the MISMA approximation (Sanchez Almeida et al. 1996) (marked by the dashed lines in Fig. 1). For very large correlation lengths (>1000 km), however, the asymmetries asymptotically converge to zero where they eventually reach - for a correlation length of approximately $10000 \mathrm{~km}$ - their macroscopic limits (complete symmetry). Figure 1 also reveals that unlike the mathematical limits, the microturbulent and macroturbulent limits are already reached numerically for finite length scales. Figure 2 shows a sample of Stokes $V$ profiles from our model calculations and displays the variation of the Stokes $V$ profile shape with the correlation length $L$. Note, in particular, the drastic change between the Stokes $V$ profiles calculated for the $L=10 \mathrm{~km}$ and $L=100 \mathrm{~km}$ case.

\subsubsection{About the microturbulent limit of photospheric structures}

Photospheric structures below $100 \mathrm{~km}$ are often considered to be optically thin and therefore have been treated in the microturbulent limit. From the latter section, however, we have seen that for a varying correlation length the shape, as well as the Stokes $V$ profile asymmetries, undergo a rapid change, in particular within the first $100 \mathrm{~km}$. When there is a preferred internal alignment e.g. by buoyancy forces the characteristic length scales of the magnetic structures in the horizontal and vertical can differ by more than an order of magnitude. This raises the question, to what extent structures below $100 \mathrm{~km}$ (measured along the LOS) can really be treated as microturbulent in (polarized) radiative transfer calculations?

From Fig. 1 we see that for a structural length scale of approximately $15 \mathrm{~km}$ the atmosphere is effectively in a microturbulent state but apparent deviations from this microturbulent limit already occur from $20 \mathrm{~km}$ upward. For a characteristic length scale of just $100 \mathrm{~km}$ both asymmetries (area and amplitude) have lost already about $50 \%$ of their initial (microturbulent) values. The rapid decrease of the area and amplitude asymmetries can be qualitatively understood from the inverse dependence of the asymmetries on the correlation length (see Sect. 3.4). The magnitude of the asymmetries is a direct consequence of the ratio of thermal absorption and statistical scattering. To quantify the degree of fluctuation and the statistical scattering occurring in the atmosphere in comparison to (regular) absorption processes we define the statistical scattering probability as follows

$P_{\text {scat }}=\frac{\kappa_{\text {stat }}}{\kappa_{\mathrm{c}}+\kappa_{l}+\kappa_{\text {stat }}}$

where the statistical scattering term $\kappa_{\text {stat }}$ is the reciprocal of the correlation length $L, \kappa_{\mathrm{c}}$ the continuous absorption coefficient at line center and $\kappa_{l}$ the line absorption coefficient. We have calculated the statistical scattering probabilities for a number of different correlation lengths, where we have used the same atmospheric condition as in the previous section. In Fig. 3 we can see the run of the statistical scattering probability over the logarithm of the optical depth for different correlation lengths. For a better illustration and a better estimate of the line forming regions we have plotted in the background of Fig. 3 the wavelength integrated mean line depression contribution function for the circular polarization (solid line). The wavelength integrated mean 

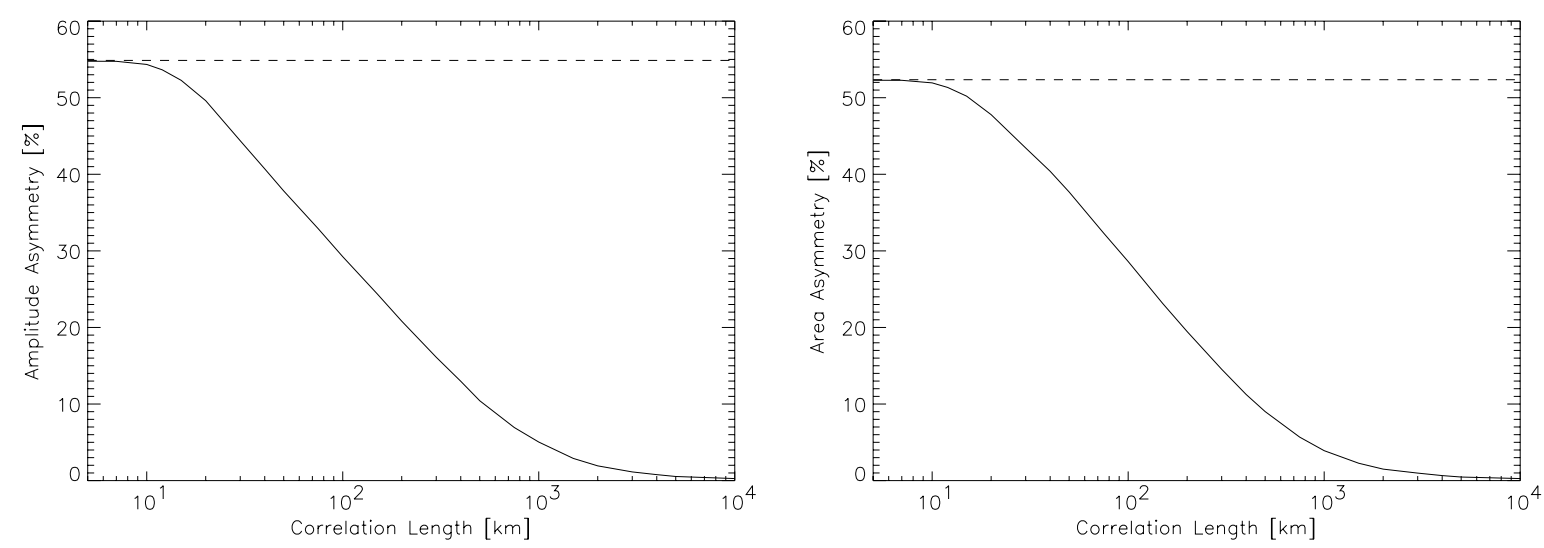

Fig. 1. The variation of the Stokes $V$ asymmetry parameters (amplitude left; area right) as a function of the correlation length $L$. The dashed lines indicate the microturbulent limit for the asymmetry parameters calculated under the MISMA approximation. Note the rapid decrease of the Stokes $V$ asymmetry with increasing correlation length.
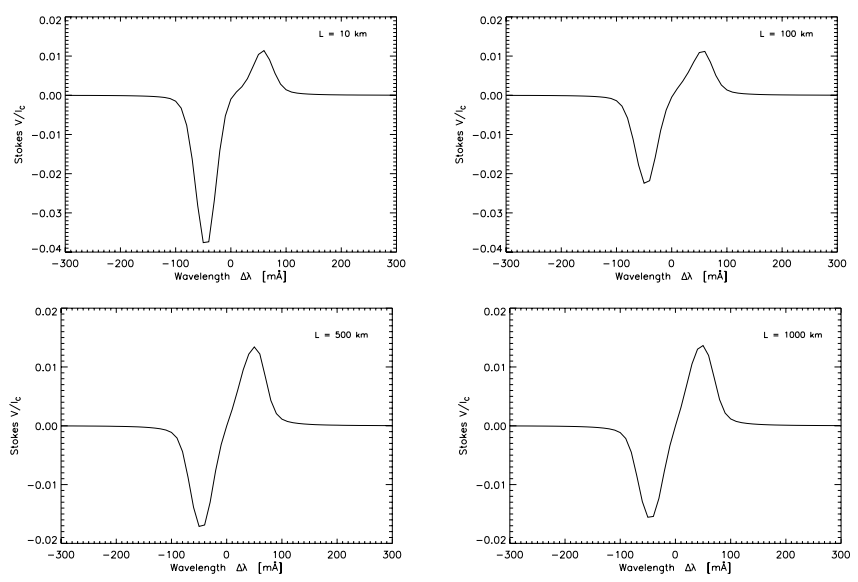

Fig. 2. Sample of Stokes $V$ profiles for a correlation length $L=10 \mathrm{~km}$ (upper left), $L=100$ (upper right), $L=500$ (lower left) and $L=$ $1000 \mathrm{~km}$ (lower right). Note, the drastic changes in profile shape as well as in area and amplitude asymmetry.

line depression contribution function for the circular polarization is an integral measure that assess the contributing layers of the emergent circular polarized signal in a stochastic atmosphere. The exact definition is given in Appendix B.

In very deep layers $\left(\log \left(\tau_{5}\right) \geq 0\right)$ of the atmosphere we see from Fig. 3 that for all correlation lengths but the smallest $(10 \mathrm{~km}$, the effective microturbulent limit) the probability that photons are statistically scattered into other regimes is very small. Most of the photons in these deep layers are subject to true absorption processes and will be thermalized before they can scatter into other atmospheric regimes. With increasing geometrical height or decreasing optical depth we see a gradual increase of the statistical scattering probability for all curves. But note that the increase of the scattering probability and the slope of the curve have a distinct dependence on the correlation length. In very high layers of the atmosphere the optical depth is so small that almost all the photons are subject only to statistical scattering regardless of their correlation length. Very interesting in that context are the statistical scattering probabilities of the $L=10 \mathrm{~km}$ (short dashed line) case and the $L=100 \mathrm{~km}$ (dashed dotted line) case. The two slopes significantly differ from each other even though both may be regarded in classical terms as microturbulent. The $L=10 \mathrm{~km}$ curve for the effective microturbulent case already indicates a significant scattering

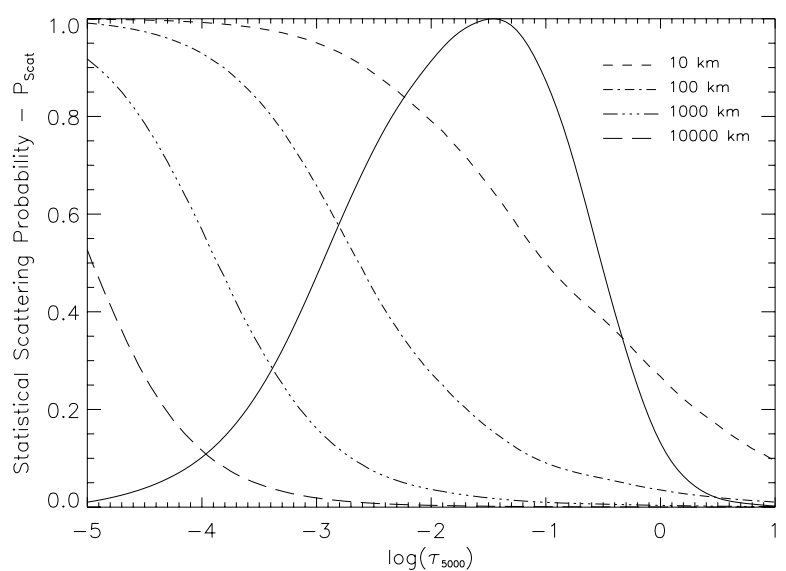

Fig. 3. The statistical scattering probability for different correlation lengths over the optical depth. In the background, the wavelength integrated mean line depression contribution function for the circular polarization is given (see explanation and definition in text).

probability for very deep layers, such that the entire line profile (wings and core) is affected by the strong statistical scattering. Whereas, the $L=100 \mathrm{~km}$ curve indicates a rather small scattering probability for the deepest line forming layers around $\log \left(\tau_{5}\right)=0$. At an optical depth where the integrated Stokes $V$ contribution function reaches its maximum, the $L=10 \mathrm{~km}$ curve has a value of approximately $70 \%$ which means that photons are much more likely to encounter a transition to another atmospheric regime (70\%) than being thermalized in their current regime. Thus, most of the photons are statistically scattered many times before they are finally thermalized in a true absorption process. At the same optical depth the scattering probability for the $L=100 \mathrm{~km}$ case is approximately $25 \%$ and therefore it has a less pronounced dependence on the statistical scattering which results in a decrease of the line asymmetry of approximately $50 \%$.

This behavior, even for a relatively moderate downflow velocity of $550 \mathrm{~m} / \mathrm{s}$, as assumed here, demonstrates that the microturbulent or microstructured (MISMA) approach does not give reliable results for atmospheric structures which exceeds the effective microturbulent limit. 

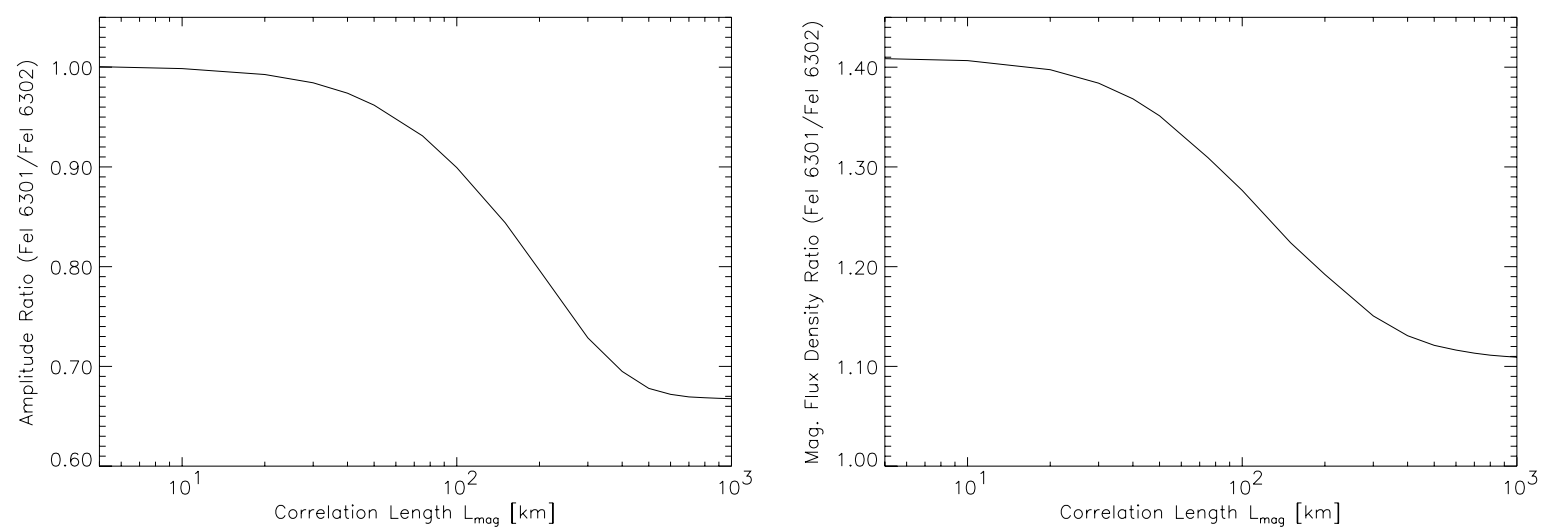

Fig. 4. The amplitude ratio $a^{6301 / 6302}$ vs. the correlation length (left) and the ratio of the longitudinal magnetic flux densities $f^{6301 / 6302}$ vs. the correlation length (right). The amplitude ratio as well as the magnetic flux ratio exhibit a clear dependence on the underlying length scale of the magnetic structures.

\subsection{A magnetopause in a mesostructured atmosphere}

In the following, we want to take a closer look on the polarized line formation in an atmosphere that comprises a magnetopause like boundary. A typical example for such a scenario might be encountered in the vicinity of a rapidly expanding large flux tube structure (Steiner 2000). Similar structures with large overlaying canopies could be recently observed in magnetohydrodynamic simulations (Schaffenberger et al. 2005) where these canopies can cover large parts of the adjacent granular cell. The scenario we have assumed here for our model calculation is intended to be a crude approximation of a possible situation encountered in the photospheric internetwork. The approximation we consider here can be conceived as an ensemble of flux tube like structures which are in pressure equilibrium with their field-free surroundings. These structures rapidly expand until the individual structures finally merge together at a certain geometrical height to form a volume filling magnetic atmosphere. The salient feature of this model is the variation of the characteristic size of the individual magnetic structures with height such that these structures evolve from small to large scale structures.

Again, we assume a simple 2-ensemble atmosphere with a number of magnetic structures which all shares the same physical conditions as well as a number of non-magnetic structures. Furthermore, we assume that the magnetic structures are in pressure equilibrium with the non-magnetic surroundings, we therefore write the following relation for the height dependence of the magnetic field strength

$B(z) \simeq B(z=0) \exp \left(-z / H_{B}\right)$,

where $H_{B}$ is the magnetic field strength scale height which is twice the gas pressure scale height and $B(z=0)$ the magnetic field strength at the geometrical height $z=0$. For $H_{B}$ we have assumed a value of $H_{B}=250 \mathrm{~km}$. The magnetic ensemble structures in our model scenario are mainly vertically orientated and we have adopted a magnetic field strength at the base $z=0$ of $B(z=0)=B(\tau=1)=500 \mathrm{G}$. For clarity and to focus on the effect of a varying correlation length, we have ignored any possible velocities in the magnetic and non-magnetic structures. Again, we have used the temperature stratification of the HOLMUL atmosphere for both ensemble structures. The merging height of the canopies (the magnetopause) is assumed to be at a geometrical height of $z \approx 450 \mathrm{~km}$ which corresponds to a logarithmic optical depth of $\log \left(\tau_{5}\right) \approx-3.65$ in the nonmagnetic HOLMUL atmosphere. At this height the field strength in the magnetic structures has already dropped to a value of approximately $80 \mathrm{G}$. For the magnetic ensemble structures we assume a fixed probability value of $1 \%$ at $z=0$. The nonmagnetic ensemble structures have a probability value of $99 \%$ at $z=0$ and we assume a fixed quasi macrostructured correlation length of $L=1000 \mathrm{~km}$ below the magnetopause for these structures. A large correlation length seems to be justified because of the much larger fill fraction of the non-magnetic structures. To mimic the rapid transition for the majority of the line-of-sights at the boundary of the magnetopause from whereon the atmosphere is assumed to be fully magnetized, we set the probability value for the magnetic structures to $100 \%$ and their correlation length to $L=1000 \mathrm{~km}$ from $\log \left(\tau_{5}\right) \approx-3.65$ on upward.

For this scenario, we have calculated synthetic Stokes spectra for the two neutral iron lines Fe I $\lambda 6301 \AA$ and $\mathrm{Fe}_{\mathrm{I}} \lambda 6302 \AA$. This is a prominent line pair which is often used for Zeeman diagnostics. Our particular interest here is to demonstrate how this prominent iron line pair depends on the length scale of the atmospheric structures below the magnetopause. For this reason we take a closer look on the differential behavior of the Stokes $V$ profiles of the two iron lines. A good measure to assess the strength of the profiles is the amplitude ratio or the line-ratio method (Stenflo 1994) which is commonly used to distinguish if the observed Stokes profiles are the result of an unresolved weak (sub-kG) or strong $(\mathrm{kG})$ magnetic field. The amplitude ratio is defined as $a^{6301 / 6302}=a\left(V^{6301}\right) / a\left(V^{6302}\right)$, where $a(V)$ represents the amplitude of the respective Stokes $V$ profile. Yet, another method going in the same direction to distinguish weak from strong magnetic field fields is the ratio of the longitudinal magnetic flux density derived from the two iron lines $f^{6301 / 6302}=B_{\text {Long }}^{6301} / B_{\text {Long }}^{6302}$ (Dominguez Cerdeña et al. 2003a). For the magnetic flux density $B_{\text {Long }}$ we have used the following approximation (see Socas-Navarro et al. 2004)

$B_{\text {Long }} \simeq \frac{C}{g_{\text {eff }}} \frac{\int_{0}^{\infty} V(\lambda) \mathrm{d} \lambda}{\int_{0}^{\infty}[\mathrm{d} I(\lambda) / \mathrm{d} \lambda] \mathrm{d} \lambda}$

where $C$ is a constant with $C=-1 /\left(4.67 \times 10^{-13} \lambda^{2}\right)$ and $g_{\mathrm{eff}}$ is the effective Landé factor of the line. The relation (74), the flux ratio $f^{6301 / 6302}$ as well as the amplitude ratio $a^{6301 / 6302}$ are direct consequences of the weak field approximation (Stenflo 1994).

Keeping all the atmospheric parameters fixed, except the correlation length of the magnetic structures below the magnetopause we have calculated the synthetic line profiles of the two iron lines for a series of correlation lengths. In Fig. 4 we have plotted the amplitude ratio $a^{6301 / 6302}$ and the longitudinal 

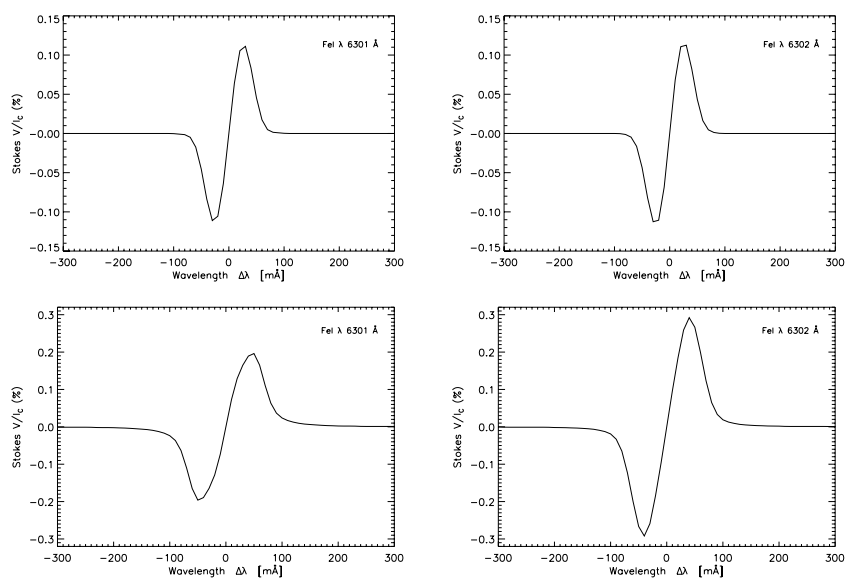

Fig. 5. The upper left and right display the Stokes $V$ profiles for the Fe I $\lambda 6301 \AA$ (left) and Fe I $\lambda 6302 \AA$ (right) line calculated with a correlation length of $L=10 \mathrm{~km}$. Both profiles in the $L=10 \mathrm{~km}$ scenario have the same amplitude and therefore would falsely indicate the presence of strong magnetic fields. The lower profiles which show the case for $L=1000 \mathrm{~km}$, exhibit the expected behavior of the profile amplitudes for an intrinsically weak field.

magnetic flux density ratio $f^{6301 / 6302}$ over the correlation length of the magnetic structures. At first glance we see a rather surprising result, despite the fact that the magnetic field strength is intrinsically weak $(B(z=0)=500 \mathrm{G}$ ) (and even rapidly decreasing with height) the amplitude as well as the flux density ratio suggest for small correlation lengths a strong underlying magnetic field in the kilo-Gauss range. The ratio for the Stokes $V$ amplitudes shows a value of 1.0 for small correlation lengths, although we would expect for our intrinsically weak field that the amplitude ratio is similar to the ratio of the corresponding Landé factors of the two iron lines which is approximately 0.67 (Solanki 1993). Moreover, one would not expect a dependence on the underlying length scale. As the magnetic flux density is also retrieved from the weak field approximation we also expect for the flux density ratio a value close to unity regardless of the correlation length. Our results show that this is not the case. For a small correlation length the flux density ratio converges to a value of 1.41 while for a correlation length, $L=1000 \mathrm{~km}$, a flux density ratio of 1.1 is obtained. It is obvious that with increasing correlation length the amplitude as well as the magnetic flux density ratio decrease. For a correlation length of approximately $300 \mathrm{~km}$ the value of the amplitude ratio and the flux density ratio converge to their (expected) weak field values. On the other hand, we see that for structures which have correlation lengths smaller than $100 \mathrm{~km}$ significant deviations from the expected values occur. Once again, we see that the underlying correlation length - the characteristic length scale of the magnetic structures - has a decisive impact on the polarized radiative transfer and the resulting Stokes $V$ profiles. To better illustrate the drastic profile variation we have plotted the Stokes $V$ profiles for the two iron lines Fe I $\lambda 6301 \AA$ and Fe I $\lambda 6302 \AA$ in Fig. 5 for a correlation length $L=10 \mathrm{~km}$ (upper row) and a correlation length $L=1000 \mathrm{~km}$ (lower row). This counterintuitive behavior of the amplitude and flux density ratios can be understood in terms of the MESMA approximation. Figure 6 shows the wavelength integrated mean line depression contribution function of the Stokes $V$ profiles $\tilde{C}_{R ; \mathrm{CP}}$ (see Appendix (B) for the two iron lines. These stochastic contribution functions $\tilde{C}_{R ; \mathrm{CP}}$ tell us where in the fluctuating atmosphere the main contributions to the Stokes $V$ signals originate. We immediately recognize the distinct shift in the contributing layers of the Stokes $V$ signal. Figure 6 reveals that for a characteristic length scale of $L=10 \mathrm{~km}$ both iron lines receive their major contributions predominantly from above the magnetic canopy which is $\operatorname{located}$ at $\log \left(\tau_{5}\right) \approx-3.65$. Whereas, for a mean length scale of $L=1000 \mathrm{~km}$ the main contributions almost exclusively come from below the magnetopause. The reduced contribution in the lower layers for both iron lines in the $L=10 \mathrm{~km}$ scenario, which is even more pronounced for the $\mathrm{Fe}_{\mathrm{I}} \lambda 6302 \AA$ line, is a direct consequence of the absorption of polarized line photons in the non-magnetic layers. The non-magnetic structures do not only fill a substantial horizontal fraction of $99 \%$ of the resolution volume, they also have, and this is even more important, a larger correlation length and therefore once photons are statistically scattered into these field-free structures, they have only a very small chance of being backscattered into a magnetic structure. Structures with large correlation length are only in weak statistical contact with their surrounding. Whether photons are being scattered into, or originate from a non-magnetic structure, the likelihood for staying in the non-magnetic regime for the rest of their trajectory is much higher for the photons than making another transition to a magnetic structure. As the scattering probability is very high in the magnetic structures (for low correlation lengths), many of the photons carrying the polarized information are scattered into the non-magnetic regimes where a substantial number of them is absorbed due to the increased opacity in the non-magnetic structures. This absorption of polarized photons in the non-magnetic structures is the main cause for the reduction of the contribution in lower layers. Beginning from the magnetopause the atmosphere is magnetically coherent (essentially macro-structured) with a probability value of $100 \%$ and a correlation length of $L=1000 \mathrm{~km}$. Although the contribution to the Stokes $V$ signal from these high layers is very small in absolute terms, it is still significant compared to the strongly reduced contribution from below the magnetopause. Hence, the absorption below the magnetopause is so efficient that the small contributions from above the magnetopause outweighs the contribution from below.

The situation is quite different for magnetic structures with larger correlation lengths. Although the probability value of the magnetic structures below the magnetopause is still only $1 \%$ the enhancement of the correlation length leads to a decrease of the statistical scattering from the magnetic structures into the nonmagnetic ones. Hence, less photons are being scattered into the denser non-magnetic structures and more polarized line photons are able to stay for their entire trajectory through the atmosphere in the same magnetic structure. The increasing coherency of the individual magnetic structures leads to the more regular shaped form of the contribution functions (normal line formation) for the $L=1000 \mathrm{~km}$ case. But, what is the cause of the unusual line ratios for small correlation lengths? Now, as we have understood why there is such a significant difference in the contributing layers with respect to the correlation length, let us turn to the question of what causes the small but decisive difference in the contribution functions for the two iron lines. From Fig. 6, we see that, although the two contribution functions for the two iron lines in the $L=10 \mathrm{~km}$ case show qualitatively the same behavior, there is a notable difference between both contribution functions. The contribution function for the Fe I $\lambda 6301 \AA$ line (dashed line) shows a larger contribution in the layer above the magnetopause than that of the Fe I $\lambda 6302 \AA$ line (solid line). As both lines in the $L=10 \mathrm{~km}$ case receive their main relative contributions from above the magnetopause the formation height of the two iron 

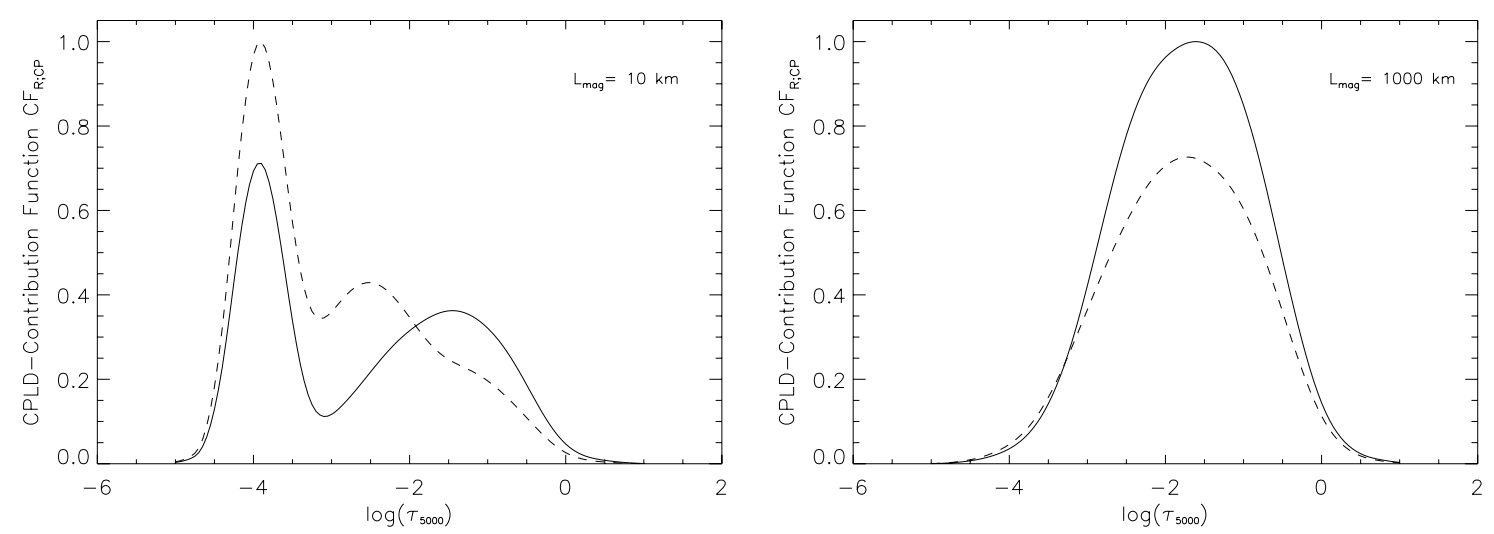

Fig. 6. The wavelength integrated mean line depression contribution function over the optical depth for the Fe $\mathrm{I} \lambda 6301 \AA$ (dashed lines) and Fe I $\lambda 6302 \AA$ line (solid lines) for a correlation length of $L=10 \mathrm{~km}$ (left) and for $L=1000 \mathrm{~km}$ (right). Note, the drastic changes of the contribution functions for both lines simply by varying the correlation length. For a correlation length of $L=10 \mathrm{~km}$ the contributions to the Stokes $V$ signal come predominantly from above the magnetopause. Note, the enhanced contribution for the Fe I $\lambda 6301 \AA$ line in the $L=10 \mathrm{~km}$ case (left). In the $L=1000 \mathrm{~km}$ case (right) one sees that for both iron lines the main contributions come from below the magnetopause.

lines play an even more important role than in the unstructured and coherent (e.g. macrostructured) case. The larger contribution of the $\mathrm{Fe}_{\mathrm{I}} \lambda 6301 \AA$ line is exclusively due to its higher formation height compared to the $\mathrm{Fe}$ I $\lambda 6302 \AA$ line. This difference in the formation height which was also illustrated and explained in terms of response functions by Martínez et al. (2006) as well as the above explained pronounced importance of the contributions from the higher layers above the magnetopause, lead to the effect that the Stokes $V$ signal of the $\mathrm{Fe}_{\mathrm{I}} \lambda 6301 \AA$ line receives an absolute larger contribution than the Fe I $\lambda 6302 \AA$ line. As the characteristic length scale of the magnetic structures grows this effect loses its significance because the peak of the contribution function begins to shift downward below the magnetopause and reduces the overemphasized difference in the formation height. This demonstrates once more that the analysis of magnetic field strengths based on the iron line pair at $6300 \AA$ in a structured magnetic atmosphere must be regarded with caution, as was also pointed out recently by Martínez et al. (2006).

\subsection{A quiet sun magnetic field strength distribution}

In the recent years the possible role of the so called internetwork magnetic field has become increasingly appreciated. Despite its elusive character, the ubiquity of these weak flux fields could be observed in a number of high spatial and high sensitivity spectropolarimetric measurements (Sigwarth et al. 1999; Lin \& Rimmele 1999; Sigwarth 2001; Lites 2002; Dominguez Cerdeña et al. 2003a; Khomenko et al. 2003; Socas-Navarro et al. 2004). The significance of these fields for the solar atmosphere is not yet clear, but the mere fact that these fields cover most of the solar surface makes them probably an important ingredient for the structuring and dynamics of the higher layers of solar atmosphere (Schrijver \& Title 2003; Sánchez Almeida 2004). The characterization of the internetwork magnetic field in terms of empirical as well as statistical parameters from Hanle and Zeeman effect measurements is currently under debate. Recently Dominguez Cerdeña et al. (2006) proposed a set of empirical probability density functions which describe the distribution of magnetic field strength in the internetwork. They emphasize that field strength in the kilo-Gauss range, even though they have low probabilities, play the dominant role in transporting most of the magnetic energy into the upper solar atmosphere.

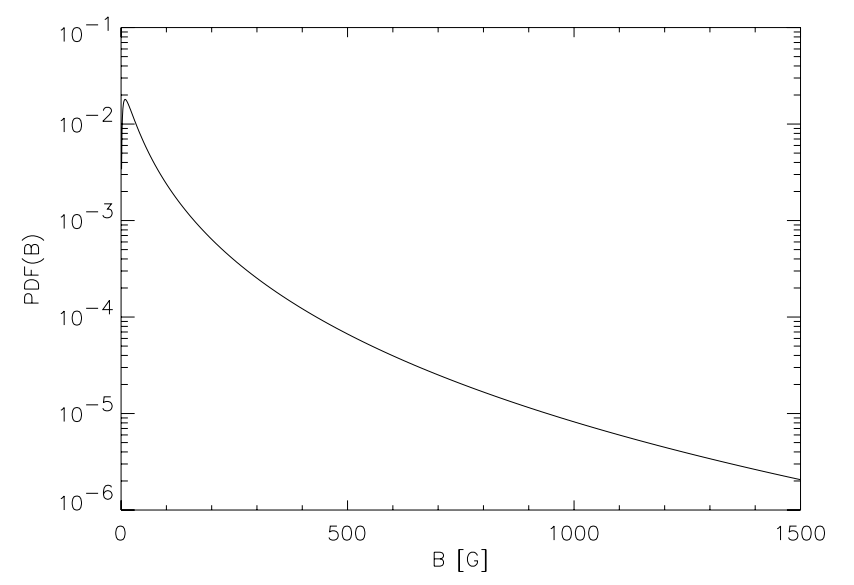

Fig. 7. Empirical probability density function for the magnetic field strength.

For empirical estimates of these pdf's from spectropolarimetric observations it is important to realize that line formation is an inherent three dimensional process and it is therefore crucial to take into account the horizontal as well as the vertical structuring of the atmosphere. In the following simple numerical model calculation we want to use the lognormal probability distribution function proposed by Dominguez Cerdeña et al. (2006) to demonstrate that the structural length scale of the individual magnetic structures has an important impact on the resulting Stokes signal and that empirical probability density functions are strongly biased by the underlying model assumptions.

The following probability density for the magnetic field strength is used

$p(B)=\frac{1}{\sqrt{\pi} \sigma B} \exp \left[-\frac{\left(\ln B-\ln B_{0}\right)^{2}}{\sigma^{2}}\right]$.

The parameters $B_{0}$ and $\sigma$ are related to the first and second order moments respectively. We have adopted the values $\sigma=1.7$ and $B_{0}=38 \mathrm{G}$ (see Dominguez Cerdeña et al. 2006). The probability density function (75) for the magnetic field strength is shown in Fig. 7, note, that the probability for a field strength larger than $1000 \mathrm{G}$ is only approximately $0.33 \%$. It is now crucial to realize that this empirical probability density function comprises a broad range of different field strength regimes, a weak 

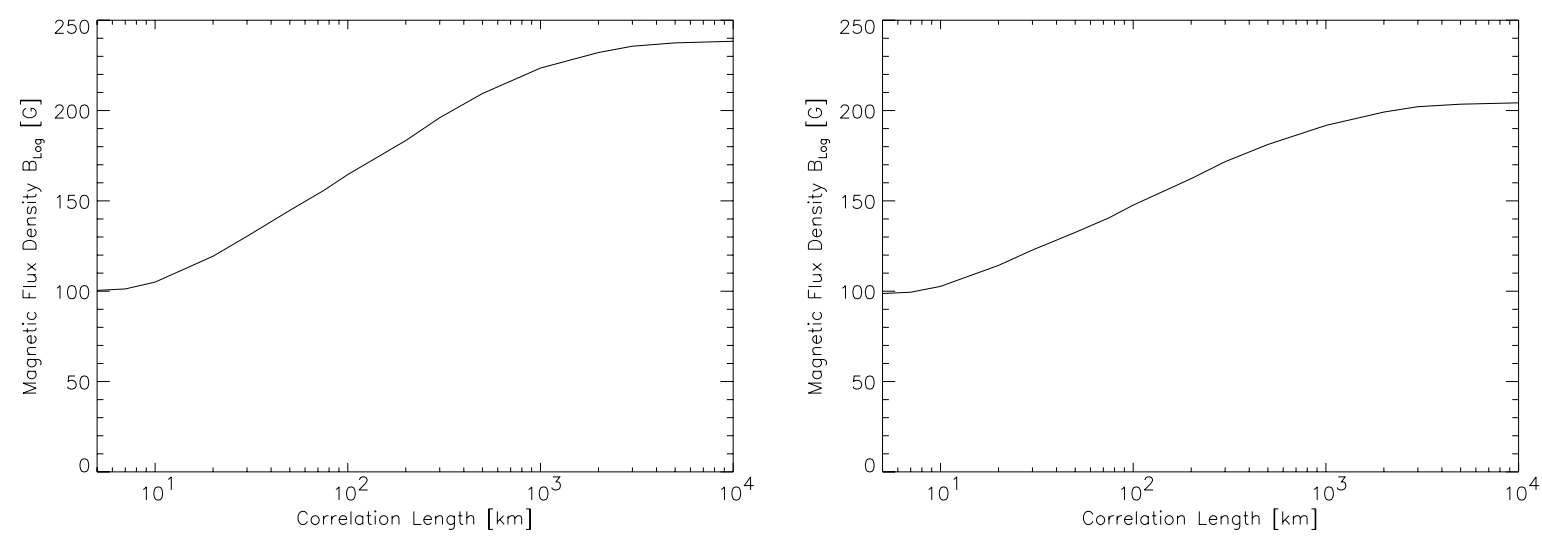

Fig. 8. The longitudinal magnetic flux density as a function of the strong field correlation length, derived from the iron line Fe I $\lambda 6301 \AA$ (left) and from the $\mathrm{Fe} \mathrm{I} \lambda 6302 \AA$ line $($ right $)$.

and probably purely turbulent range as suggested by Hanle effect measurements, a wide range of intermediate field strengths which are approximately in equipartition to their non-magnetic surroundings and a strong field range which is assumed to be populated by small intense kilo-Gauss structures. This picture is also supported by an increasing evidence for the existence of a small scale mixture of weak, intermediate and strong fields in a single resolution element (Socas-Navarro \& Lites 2004).

But do these different field regimes all have the same characteristic length scale? As magnetic buoyancy keeps stronger magnetic field structures more aligned to the vertical direction than weaker field structures, the extent of the line-of-sight which traverses through an individual magnetic structure cannot be the same for all field strengths. There must be a dependence of the correlation length on the field strength. To demonstrate the importance of this effect, we assume the following simplified scenario: for field structures below one kilo-Gauss field strength we adopt a correlation length of $10 \mathrm{~km}$ (effectively microturbulent) and for structures with field strength larger than one kilo-Gauss we vary the correlation length in a range from 10 to $10000 \mathrm{~km}$. Again, we have synthesized the two iron lines at $\lambda 6300 \AA$ and have used the temperature stratification of the HOLMUL atmosphere. To assess and quantify the influence of the correlation length on the Stokes $V$ profiles, we have used the longitudinal magnetic flux density (74), which gives us an estimate of the magnitude of the circular polarized signal.

Figure 8 shows how the magnetic flux densities vary with the correlation length of the strong field structures. For both iron lines one can see from Fig. 8 the rapid increase of the flux density with increasing correlation length. Please note, that no other parameter but the correlation length of the kilo-Gauss structures has been changed in our scenario, the probability density function in our calculation is for all correlation lengths the same. For very small structures $(\sim 10 \mathrm{~km})$ the magnetic flux density saturates and converges into the microturbulent limit which corresponds to the value calculated for the probability density function (75) under MISMA approximation. For very large structures $(\geq 1000 \mathrm{~km})$ the value for the magnetic flux density converges into the macroturbulent limit. We see that just by increasing the correlation length of the strong field structures from micro-scales to macro-scales the magnetic flux densities (and, hence, the circular polarizations of both iron lines) are more than doubled. This, again, demonstrates how drastic the underlying length scale of the magnetic structures affects the process of polarized line formation. Although the underlying probability density function (75) has not changed, the effect of the strong field structures is more and more pronounced simply by increasing their correlation lengths. The growing correlation length is responsible for the additional magnetic flux derived from both spectral lines.

Another intriguing aspect is the increasing difference of the flux densities calculated from both iron lines with increasing correlation length (Fig. 8). The flux density for the iron line $\mathrm{Fe}$ I $\lambda 6301 \AA$ seems to grow faster than that of the Fe $\mathrm{I} \lambda 6302 \AA$ line. This difference in the magnetic flux density ratio would suggest that the underlying probability density is dominated by the kiloGauss structures. This misleading effect is also highlighted by Fig. 9 which shows the amplitude ratio $r^{6301 / 6302}$ of the two iron lines as a function of the strong field correlation length. The amplitude ratio would also lead to the erroneous conclusion that the underlying probability density is strongly biased towards kiloGauss structures, even though the kilo-Gauss structures make up less than $1 \%$ of the overall distribution.

So we see again the decisive role of the correlation length and that the simple derivation of empirical probability density functions from spectropolarimetric measurements is not straight forward. There is no direct link to the number density of strong field components without accounting at the same time for the inherent length scale (correlation length) of the magnetic structures. The magnitude and the profile form of the polarized signal is therefore much more the result of the complex interplay between the horizontal probability density function and the characteristic length scale of the individual structures.

\section{Summary and conclusions}

The ubiquity as well as the degree of asymmetry of observed Stokes $V$ profiles are direct indicators of the inhomogeneous nature of the unresolved magnetic field in the solar photosphere. Stokes $V$ profile asymmetries can be explained in terms of atmospheric gradients or more general in terms of the finite spatial extent (correlation length) of the underlying magnetic field. This finite character of the individual structures is the essential starting point of the here presented concept of a mesostructured magnetic atmosphere (MESMA). In contrast to the proposed microstructured magnetic atmosphere (Sanchez Almeida et al. 1996) the MESMA approach does not rely on the assumption of a solely microturbulent magnetic field, the MESMA concept rather allows all kinds of different characteristic length scales to be present in the atmosphere.

We have put forward a very generic statistical model (Sect. 2) which is based on a Markov random field. The key idea here 


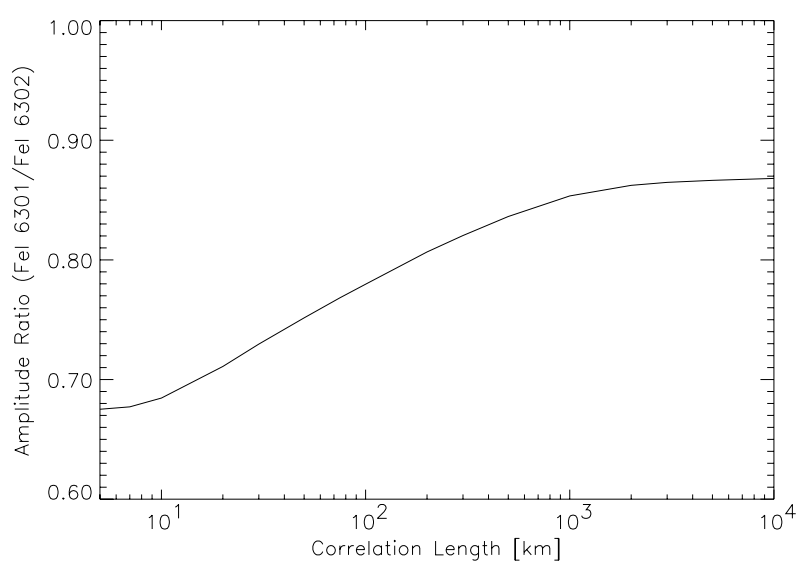

Fig. 9. The amplitude ratio between the two iron lines Fe I $\lambda 6301 \AA$ and Fe I $\lambda 6302 \AA$ as a function of the strong field correlation length. Note, that the underlying pdf is for all correlation lengths the same.

was to explicitly account for the spatial coherency of the underlying structures. This model facilitates the derivation of a stochastic transport equation for the conditional Stokes vector (Sect. 3). By utilizing a modified evolution operator we could derive in analogy to the deterministic case a formal solution for the stochastic polarized transport equation (Sect. 3.2). In Sect. 3.3 we have shown that the microstructured (MISMA) as well as the macrostructured approach are special limits of our more general mesostructured approach. Hence, the here outlined concept of a mesostructured magnetic atmosphere presents the natural extension of the MISMA approach. We could also show that asymmetric Stokes profiles are a natural consequence for an atmosphere where velocity and the magnetic fields are organized on finite scales. Moreover, our stochastic approach allowed us to determine a direct relation between the characteristic length scale of the atmospheric structures and the degree of the asymmetry (Sect. 3.4).

One of the most important parameter in the MESMA approach is the correlation length (the mean length scale) of the underlying atmospheric structures. As the correlation length appears explicitly in the stochastic transfer equation for polarized light it can be used as a diagnostic parameter. In a number of numerical experiments we used exactly this parameter to demonstrate the importance of the correlation length for the polarized line formation.

In a first numerical experiment (Sect. 4.2) we have analyzed the line formation in a fluctuating atmosphere which is characterized by two kinds (ensembles) of different structures (magnetic and non-magnetic). Albeit this is a commonly invoked scenario for the interpretation of unresolved magnetic structures our approach fully accounts for the lack of knowledge about the underlying organization and geometry. In these model calculations it could be demonstrated that the Stokes $V$ profiles as well as the amplitude and area asymmetries have a clear functional dependence upon the correlation length of the underlying structures. We also showed (Sect. 4.2.1) that the effective microturbulent limit is already reached for a finite correlation length of approximately $15 \mathrm{~km}$. On the other hand this means that for correlation lengths larger than $20 \mathrm{~km}$ the atmosphere can no longer be described in terms of a microstructured magnetic atmosphere.

An atmosphere which is characterized by an extended magnetopause like structure was investigated in Sect. (4.3). Again, our results demonstrate the distinct change of the Stokes $V$ profiles and other derived quantities with varying correlation length.
The line formation strongly depends on the structure below the magnetopause. The ratio of the Stokes $V$ profile amplitude and the ratio of the magnetic flux density for the two iron line Fe I $\lambda 6301 \AA$ and $\mathrm{Fe}$ I $\lambda 6302 \AA$ are significantly altered just by changing the characteristic length scale of the magnetic field. These ratios falsely indicate for small correlation lengths the presence of strong kilo-Gauss magnetic fields, although the entire model atmosphere is intrinsically weak. Thus, the validity and application of these ratios as indicators for strong magnetic field structures is highly questionable in inhomogeneous atmospheres.

In Sect. (4.4) we finally investigated the polarized spectral signature which originated from a field strength distribution given by a recently derived empirical probability density functions. We placed particular emphasis on the fact that the underlying magnetic field cannot properly be described in the context of a field strength distribution without taking into account the characteristic length scale of the individual structures. As the field strength covers a broad range from very weak to strong fields, the characteristic length scale of these structures has to be different. If we realistically assume that strong field structures possess intrinsic length scales that are larger than that of the weaker field structures we have shown that the strong field part of the distribution dominates in contributing to the resulting Stokes $V$ profile and would lead to an overestimation of the strong field structures. Therefore neglecting the correlation length (line-of-sight filling factor) of the underlying structures will lead to incorrect estimates for the magnetic field and flux distribution.

The here introduced concept of a mesostructured magnetic atmosphere (MESMA) is a statistical attempt to cope with the underlying complexity of the magnetic field in the solar photosphere in terms of polarized radiative transfer modeling. As the majority of the magnetic flux in the solar photosphere is still not resolved and the fundamental length scale of the magnetic structures is unknown, the here presented stochastic approach offers a viable and promising tool for the interpretation of spectropolarimetric observations and the diagnostics of photospheric magnetic fields.

First Stokes profile inversions on the basis of the MESMA approximation were made by Carroll \& Staude (2005b, 2006); Carroll (2007). They have demonstrated the feasibility of their approach which allowed them to estimate the characteristic length scale of internetwork and penumbral magnetic field structures.

Acknowledgements. We would like to thank the referee B. Ruiz Cobo for many helpful and constructive comments and suggestions that helped to improve this paper. The authors also gratefully acknowledge financial support from the Deutsche Forschungsgemeinschaft (DFG) under the grant CA 475/1-1.

\section{References}

Auer, L. H., \& Heasley, J. N. 1978, A\&A, 64, 67

Auvergne, M., Frisch, H., Frisch, U., Froeschle, C., \& Pouquet, A. 1973, A\&A, 29, 93

Carroll, T. A. 2007, Proceedings of Solar Modern Facilities - Advanced Solar Science, ed. F. Kneer, K. G. Puschmann, \& D. Wittmann (University Press Göttingen), 297

Carroll, T. A., \& Staude, J. 2003, Astron. Nachr., 324, 392

Carroll, T. A., \& Staude, J. 2005a, Astron. Nachr., 326, 296

Carroll, T. A., \& Staude, J. 2005b, ESA SP-596: Chromospheric and Coronal Magnetic Fields

Carroll, T. A., \& Staude, J. 2006, Solar Polarization 4, ed. R. Casini, \& B. W. Lites, 19 September 2005, National Center for Atmospheric Research, Boulder, Colorado, USA, ASP Conf. Ser., 358

Domínguez Cerdeña, I., Sánchez Almeida, J., \& Kneer, F. 2003a, A\&A, 407, 741 
Domínguez Cerdeña, I., Kneer, F., \& Sánchez Almeida, J. 2003b, ApJ, 582, L55 Domínguez Cerdeña, I., Sánchez Almeida, J., \& Kneer, F. 2006, ApJ, 636, 496 Domke, H., \& Pavlov, G. G. 1979, Ap\&SS, 66, 47

Frisch, H., \& Frisch, U. 1976, MNRAS, 175, 157

Frisch, H., Sampoorna, M., \& Nagendra, K. N. 2005, A\&A, 442, 11

Frisch, H., Sampoorna, M., \& Nagendra, K. N. 2006, A\&A, 453, 1095

Gail, H. P., Hundt, E., Kegel, W. H., Schmid-Burgk, J., \& Traving, G. 1974, A\&A, 32, 65

Grossmann-Doerth, U., Schüssler, M., Sigwarth, M., \& Steiner, O. 2000, A\&A, 357,351

Gu, Y., Lindsey, C., \& Jefferies, J. T. 1995, ApJ, 450, 318

Holweger, H., \& Mueller, E. A. 1974, Sol. Phys., 39, 19

Khomenko, E. V., Collados, M., Solanki, S. K., Lagg, A., \& Trujillo Bueno, J. 2003, A\&A, 408, 1115

Landi Degl'Innocenti, E., \& Landi Degl'Innocenti, M. 1981, Nuovo Cimento B Serie, 62, 1

Landi Degl'Innocenti, E., \& Landolfi, M. 1983, Sol. Phys., 87, 221

Landi Degl'Innocenti, E., \& Landi Degl'Innocenti, M. 1985, Sol. Phys., 97, 239

Landi Degl'Innocenti, E., \& Landolfi, M. 2004, Polarization in Spectral Lines, Astrophysics and Space Science Library (Dordrecht, Boston: Kluwer Academic Publishers)

Lin, H., \& Rimmele, T. 1999, ApJ, 514, 448

Lites, B. W. 2002, ApJ, 573, 431

López Ariste, A. 2002, ApJ, 564, 379

Magnan, C. 1985 , A\&A, 144, 186

Magnus, W. 1954, Commun. Pure Appl. Math, 7, 649

Martínez González, M. J., Collados, M., \& Ruiz Cobo, B. 2006, A\&A, 456, 1159

Nikoghossian, A. G., Pojoga, S., \& Mouradian, Z. 1997, A\&A, 325, 813

Press, W. H., Teukolsky, S. A., Vetterling, W. T., \& Flannery, B. P. 1992 (Cambridge: University Press), c1992, 2nd edn.

Rees, D. E. 1987, Numerical Radiative Transfer, ed. W. Kalkofen (Cambridge: University Press), 213

Rees, D. E., \& Geers, G. 1996, Sol. Phys., 164, 103

Ruiz Cobo, B., \& del Toro Iniesta, J. C. 1994, A\&A, 283, 129

Sanchez Almeida, J. 1997, ApJ, 491, 993
Sánchez Almeida, J. 2004, The Solar-B Mission and the Forefront of Solar Physics, ASP Conf. Ser., 325, 115

Sánchez Almeida, J., \& Lites, B. W. 2000, ApJ, 532, 1215

Sanchez Almeida, J., Collados, M., \& del Toro Iniesta, J. C. 1989, A\&A, 222, 311

Sanchez Almeida, J., Landi degl'Innocenti, E., Martinez Pillet, V., \& Lites, B. W. 1996, ApJ, 466, 537

Schaffenberger, W., Wedemeyer-Böhm, S., Steiner, O., \& Freytag, B. 2005, ESA SP-596: Chromospheric and Coronal Magnetic Fields

Schrijver, C. J., \& Title, A. M. 2003, ApJ, 597, L165

Schüssler, M. 1986, Small Scale Magnetic Flux Concentrations in the Solar Photosphere, 103

Schüssler, M. 2003, Astron. Soc. Pacific Conf. Ser., 307, 601

Sigwarth, M. 2001, ApJ, 563, 1031

Sigwarth, M., Balasubramaniam, K. S., Knölker, M., \& Schmidt, W. 1999, A\&A, 349,941

Socas-Navarro, H., \& Lites, B. W. 2004, ApJ, 616, 587

Socas-Navarro, H., Martínez Pillet, V., \& Lites, B. W. 2004, ApJ, 611, 1139

Solanki, S. K. 1993, Space Sci. Rev., 63, 1

Solanki, S. K., \& Montavon, C. A. P. 1993, A\&A, 275, 283

Solanki, S. K., \& Bruls, J. H. M. J. 1994, A\&A, 286, 269

Stein, R. F., \& Nordlund, A. 1998, ApJ, 499, 914

Stein, R. F., \& Nordlund, Å. 2006, ApJ, 642, 1246

Steiner, O. 2000, Sol. Phys., 196, 245

Steiner, O., Grossmann-Doerth, U., Knoelker, M., \& Schuessler, M. 1998, ApJ, 495, 468

Stenflo, J. O. 1976, IAU Symp. 71: Basic Mechanisms of Solar Activity, 69

Stenflo, J. O. 1994, Solar magnetic fields: polarized radiation diagnostics, Astrophysics and Space Science Library (Dordrecht, Boston: Kluwer Academic Publishers)

Trujillo Bueno, J., Shchukina, N., \& Asensio Ramos, A. 2004, Nature, 430, 326 van Kampen, N. G. 1992, Stochastic Processes in Physics and Chemistry (Revised and enlarged edition) (Amsterdam: Elsevier Science)

Vögler, A., \& Schüssler, M. 2003, Astron. Nachr., 324, 399

Vögler, A., Shelyag, S., Schüssler, M., et al. 2005, A\&A, 429, 335 
T. A. Carroll and M. Kopf: The meso-structured magnetic atmosphere, Online Material $p 1$

\section{Online Material}




\section{Appendix A: The master equation for the mesostructured magnetic atmosphere}

In following we want to derive a differential equation (master equation) that governs the spatial evolution of the probability density along the spatial coordinate $s$. To derive such a master equation for the Kubo-Andreson process let us take the Taylor expansion of the transition probability (12) at the coordinate $s^{\prime}$ which gives

$$
\begin{aligned}
p\left(\boldsymbol{B}^{\prime \prime}, s^{\prime}+\Delta s \mid \boldsymbol{B}^{\prime}, s^{\prime}\right)= & \left(1-\gamma\left(\boldsymbol{B}^{\prime}\right) \Delta s\right) \delta\left(\boldsymbol{B}^{\prime \prime}-\boldsymbol{B}^{\prime}\right) \\
& +\gamma\left(\boldsymbol{B}^{\prime}\right) p\left(\boldsymbol{B}^{\prime \prime}\right) \Delta s^{\prime}+O\left(\Delta s^{\prime 2}\right),
\end{aligned}
$$

where we have assumed that $\gamma\left(\boldsymbol{B}^{\prime}\right)$ is sufficiently small compared to $\Delta s$ such that second and higher order terms of the exponential are negligible. We then introduce the transition rate $w\left(\boldsymbol{B}^{\prime \prime}, \boldsymbol{B}^{\prime}\right)$ which describes the probability per unit length scale for a transition from an atmospheric regime $\boldsymbol{B}^{\prime}$ to a regime $\boldsymbol{B}^{\prime \prime}$. The transition rate can be obtained from the spatial derivative of the KuboAnderson Process (12), hence, for a sufficiently small $\gamma(\boldsymbol{B})$ the transition rate directly follows from Eq. (A.1),

$w\left(\boldsymbol{B}^{\prime \prime}, \boldsymbol{B}^{\prime}\right)=\gamma\left(\boldsymbol{B}^{\prime}\right) p\left(\boldsymbol{B}^{\prime \prime}\right)$.

To introduce the dynamics of the process, we use the ChapmanKolmogorov Eq. (11),

$p\left(\boldsymbol{B}^{\prime \prime}, s^{\prime}+\Delta s \mid \boldsymbol{B}, s\right)=$

$$
\int p\left(\boldsymbol{B}^{\prime \prime}, s^{\prime}+\Delta s \mid \boldsymbol{B}^{\prime}, s^{\prime}\right) p\left(\boldsymbol{B}^{\prime}, s^{\prime} \mid \boldsymbol{B}, s\right) \mathrm{d} \boldsymbol{B}^{\prime},
$$

and replace the first factor under the integral on the r.h.s. with Eq. (A.1) to obtain

$p\left(\boldsymbol{B}^{\prime \prime}, s^{\prime}+\Delta s \mid \boldsymbol{B}, s\right)=\left(1-\gamma\left(\boldsymbol{B}^{\prime \prime}\right) \Delta s\right) p\left(\boldsymbol{B}^{\prime}, s^{\prime} \mid \boldsymbol{B}, s\right)$

$+\Delta s \int w\left(\boldsymbol{B}^{\prime \prime}, \boldsymbol{B}^{\prime}\right) p\left(\boldsymbol{B}^{\prime}, s^{\prime} \mid \boldsymbol{B}, s\right) \mathrm{d} \boldsymbol{B}^{\prime}$,

Please note, that we have already performed the integration of the first term over the delta function with respect to $\boldsymbol{B}^{\prime}$. We rearrange Eq. (A.4) to write

$\frac{p\left(\boldsymbol{B}^{\prime \prime}, s^{\prime}+\Delta s \mid \boldsymbol{B}_{s}, s\right)-p\left(\boldsymbol{B}^{\prime \prime}, s^{\prime} \mid \boldsymbol{B}_{s}, s\right)}{\Delta s}=$

$-\gamma\left(\boldsymbol{B}^{\prime \prime}\right) p\left(\boldsymbol{B}^{\prime \prime}, s^{\prime} \mid \boldsymbol{B}, s\right) \Delta s$

$+\Delta s \int w\left(\boldsymbol{B}^{\prime \prime}, \boldsymbol{B}^{\prime}\right) p\left(\boldsymbol{B}^{\prime}, s^{\prime} \mid \boldsymbol{B}, s\right) \mathrm{d} \boldsymbol{B}^{\prime}$.

The definition of the transition rate (A.2) allows us to write the fluctuation rate $\gamma\left(\boldsymbol{B}^{\prime \prime}\right)$ in the following form,

$\gamma\left(\boldsymbol{B}^{\prime \prime}\right)=\int w\left(\boldsymbol{B}^{\prime}, \boldsymbol{B}^{\prime \prime}\right) \mathrm{d} \boldsymbol{B}^{\prime}$.

This relation can now be used to write (A.5) as

$\frac{p\left(\boldsymbol{B}^{\prime \prime}, s^{\prime}+\Delta s \mid \boldsymbol{B}_{s}, s\right)-p\left(\boldsymbol{B}^{\prime \prime}, s^{\prime} \mid \boldsymbol{B}_{s}, s\right)}{\Delta s}=$

$+\Delta s \int w\left(\boldsymbol{B}^{\prime \prime}, \boldsymbol{B}^{\prime}\right) p\left(\boldsymbol{B}^{\prime}, s^{\prime} \mid \boldsymbol{B}, s\right) \mathrm{d} \boldsymbol{B}^{\prime}$

$-\Delta s \int w\left(\boldsymbol{B}^{\prime}, \boldsymbol{B}^{\prime \prime}\right) p\left(\boldsymbol{B}^{\prime \prime}, s^{\prime} \mid \boldsymbol{B}, s\right) \mathrm{d} \boldsymbol{B}^{\prime}$.

Taking the limit as $\Delta s \rightarrow 0$ gives the differential equation which describes the spatial evolution of the conditional probability density,

$$
\begin{aligned}
\frac{\partial p\left(\boldsymbol{B}^{\prime \prime}, s^{\prime} \mid \boldsymbol{B}, s\right)}{\partial s^{\prime}}= & \int w\left(\boldsymbol{B}^{\prime \prime}, \boldsymbol{B}^{\prime}\right) p\left(\boldsymbol{B}, s^{\prime} \mid \boldsymbol{B}, s\right) \mathrm{d} \boldsymbol{B}^{\prime} \\
& -\int w\left(\boldsymbol{B}^{\prime}, \boldsymbol{B}^{\prime \prime}\right) p\left(\boldsymbol{B}^{\prime \prime}, s^{\prime} \mid \boldsymbol{B}, s\right) \mathrm{d} \boldsymbol{B}^{\prime}
\end{aligned}
$$

To obtain the differential equation for the unconditional probability density function we multiply (A.8) with $p(\boldsymbol{B}, s)$ followed by an integration over the entire state space $\boldsymbol{B}$. After rearranging the indices we finally yield the master equation for the KuboAnderson process,

$$
\begin{aligned}
\frac{\partial p(\boldsymbol{B}, s)}{\partial s}= & \int w\left(\boldsymbol{B}, \boldsymbol{B}^{\prime}\right) p\left(\boldsymbol{B}^{\prime}, s^{\prime}\right) \mathrm{d} \boldsymbol{B}^{\prime} \\
& -\int w\left(\boldsymbol{B}^{\prime}, \boldsymbol{B}\right) p(\boldsymbol{B}, s) \mathrm{d} \boldsymbol{B}^{\prime} .
\end{aligned}
$$

The discrete analogue of the master equation is given by

$$
\begin{aligned}
\frac{\partial p(\boldsymbol{B}, s)}{\partial s}= & \sum_{B^{\prime}} w\left(\boldsymbol{B}, \boldsymbol{B}^{\prime}\right) p\left(\boldsymbol{B}^{\prime}, s^{\prime}\right) \\
& -\sum_{B^{\prime}} w\left(\boldsymbol{B}^{\prime}, \boldsymbol{B}\right) p(\boldsymbol{B}, s),
\end{aligned}
$$

where the sum spans over the entire discrete state space of $\boldsymbol{B}^{\prime}$.

\section{Appendix B: The wavelength integrated mean line depression contribution function for circular polarized light}

The wavelength integrated mean line depression contribution function can be derived from the formal solution of the mean conditional Stokes vector (44) which reads for a semi-infinite atmosphere

$\boldsymbol{Y}_{\boldsymbol{B}}(0)=\int_{0}^{\infty} \tilde{\boldsymbol{O}}_{B}(0, \tau) \tilde{\boldsymbol{j}}_{\boldsymbol{B}}(\tau) \mathrm{d} \tau$

where $\tilde{\boldsymbol{j}}_{\boldsymbol{B}}(\tau)$ is the modified conditional line emission vector (37). From the formal solution we can define the contribution function to the emergent mean conditional Stokes vector as

$\tilde{\boldsymbol{C}}_{\boldsymbol{B}}(\log \tau)=\ln (10) \tau \tilde{\boldsymbol{O}}_{B}(0, \tau) \tilde{\boldsymbol{j}}_{\boldsymbol{B}}(\tau)$.

To derive the line depression contribution function of the conditional Stokes vector we introduce the line depression of the conditional Stokes vector as $\mathbf{R}=\mathbf{1}-\frac{\boldsymbol{Y}}{\left\langle I_{\mathrm{c}}\right\rangle}$. The derivation of the line depression contribution function is then formally identical to the non-stochastic case (see e.g. Solanki \& Bruls 1994; Rees $\&$ Geers 1996). By using again the formal solution (B.1) we can write for the line depression contribution function of the mean conditional Stokes vector

$\tilde{\boldsymbol{C}}_{\boldsymbol{B} \boldsymbol{R}}(\log \tau)=\ln (10) \tau \tilde{\boldsymbol{O}}_{B}(0, \tau) \frac{\left\langle I_{\mathrm{c}}(\tau)\right\rangle-\tilde{\boldsymbol{j}}_{\boldsymbol{B}}(\tau)}{\left\langle I_{\mathrm{c}}(0)\right\rangle}$

The wavelength integrated mean line depression contribution function of the circular polarization can then finally be defined as

$\tilde{C}_{R ; \mathrm{CP}}(\log \tau)=\int_{\Lambda} \int_{\hat{\boldsymbol{B}}}\left|\tilde{C}_{B R ; V}(\boldsymbol{B}, \lambda, \tau)\right| p(\boldsymbol{B}) \mathrm{d} \boldsymbol{B} \mathrm{d} \lambda$,

where the integration of the inner integral is performed over the state space $\hat{\boldsymbol{B}}$ and the outer integration over the wavelength domain $\Lambda$ of the spectral line. 